\title{
A Bombieri-Vinogradov theorem for all number fields
}

\author{
M. Ram Murty and Kathleen L. Petersen
}

January 30,2012

\begin{abstract}
The classical theorem of Bombieri and Vinogradov is generalized to a non-abelian, non-Galois setting. This leads to a prime number theorem of "mixed-type" for arithmetic progressions "twisted" by splitting conditions in number fields. One can view this as an extension of earlier work of M. R. Murty and V. K. Murty on a variant of the Bombieri-Vinogradov theorem. We develop this theory with a view to applications in the study of the Euclidean algorithm in number fields and arithmetic orbifolds.
\end{abstract}

Dirichlet's density theorem gives an asymptotic estimate for the density of primes in arithmetic progressions. Let $\pi(x)$ denote the number of primes $p \leq x$, and for positive integers $a \leq q$ such that $(a, q)=1$, denote by $\pi(x, q, a)$ the number of primes $p \leq x$ which satisfy the congruence $p \equiv a(\bmod q)$. Dirichlet's theorem indicates that as $x \rightarrow \infty$

$$
\pi(x, q, a) \sim \frac{\pi(x)}{\phi(q)}
$$

where $\phi$ is Euler's totient function, and $f \sim g$ means that $f / g \rightarrow 1$. The Riemann hypothesis for all Dirichlet $L$-functions implies that the error term satisfies the estimate

$$
\left|\pi(x, q, a)-\frac{\pi(x)}{\phi(q)}\right| \ll x^{\frac{1}{2}} \log q x,
$$

where $f \ll g$ (equivalently $f=O(g)$ ) means that $|f / g|$ is bounded, and will be referred to by saying that $f$ is of order $g$. The celebrated theorem of Bombieri [3] and Vinogradov [24] shows that this estimate holds on the average.

Theorem 0.1 (Bombieri, Vinogradov). Let $A>0$ be given. Then there is a $B=B(A)>0$ so that for $Q=x^{\frac{1}{2}}(\log x)^{-B}$

$$
\sum_{q \leq Q} \max _{(a, q)=1} \max _{y \leq x}\left|\pi(y, q, a)-\frac{\pi(y)}{\phi(q)}\right| \ll \frac{x}{(\log x)^{A}}
$$

In [17], a variant of Theorem 0.1 is proven for $K / \mathbb{Q}$ a Galois extension of number fields. The goal of this paper is to prove an analogous theorem without the Galois assumption. Specifically, let $K$ be a number field and $M$ a subfield of $K$ (possibly $M=K$ ) such that $K / M$ is Galois. Let $G=\operatorname{Gal}(K / M)$ and let $C$ be a conjugacy class in $G$. Let $\mathfrak{p}$ be a prime ideal of $M$ unramified in $K$ and let $\sigma_{\mathfrak{p}}$ denote the conjugacy class of Frobenius automorphisms corresponding to prime ideals $\mathfrak{q}$ of $K$ lying over $\mathfrak{p}$. Define $\pi(x, C)$ to be the number of prime ideals $\mathfrak{p}$ of $M$ unramified in $K$ with $\sigma_{\mathfrak{p}}=C$ and $N \mathfrak{p} \leq x$ where $N=N_{M / \mathbb{Q}}$. With $a, q$ as above let $\pi(x, C, q, a)$ denote the number of primes ideals $\mathfrak{p}$ of $M$ unramified in $K$ with $\sigma_{\mathfrak{p}}=C, N \mathfrak{p} \leq x$ and $N \mathfrak{p} \equiv a(\bmod q)$. By the Chebotarev density theorem [23]

$$
\pi(x, C, q, a) \sim d(C, q, a) \pi(x)
$$

for a density $d(C, q, a) \geq 0$. If $K \cap \mathbb{Q}\left(\zeta_{q}\right)=\mathbb{Q}$ where $\zeta_{q}$ is a primitive $q$-th root of unity, then

$$
d(C, q, a)=\frac{|C|}{|G|} \frac{1}{\phi(q)} .
$$

We prove the following theorem, which is equivalent to Theorem 4.1 proven in $\S 4$. 
Theorem 0.2. Let $K / M$ be a Galois extension of number fields, with group $G$ and let $C$ be a conjugacy class in $G$. Let $H$ be the largest abelian subgroup of $G$ such that $H \cap C \neq \emptyset$ and let $E$ be the fixed field of $H$. Let $\eta=\max \{[E: \mathbb{Q}]-2,2\}$ and for any $\epsilon>0$ let $Q=x^{\frac{1}{\eta}-\epsilon}$. Then for any $A>0$,

$$
\sum_{q \leq Q}^{\prime} \max _{(a, q)=1} \max _{y \leq x}|\pi(y, C, q, a)-d(C, q, a) \pi(y)| \ll \frac{x}{(\log x)^{A}}
$$

where the decoration' indicates that the summation is over only those $q$ such that $K \cap \mathbb{Q}\left(\zeta_{q}\right)=\mathbb{Q}$.

In comparison with Theorem 0.1, a value of $\eta=2$ would be ideal, but is not within the grasp of current methods. The coarsest interpretation of our results yields an $\eta$ value of $\max \{[K: \mathbb{Q}]-2,2\}$.

There are several motivations for deriving this theorem. One of them is to the study of the Euclidean algorithm in number fields as developed in [12]. Another is to the study of arithmetic orbifolds dealing with the structure of quotients of $P S L_{2}\left(\mathcal{O}_{K}\right)$ as in [18]. In many applications we have in mind, this theorem will be used in conjunction with the lower bound sieve, where it is often required to produce infinitely many primes $p$ with a certain splitting type in a fixed (not necessarily Galois) extension with some restriction on the prime divisors of $p-1$. We will not pursue any of these applications in this paper. Our purpose here is to derive the result with all of the technical details clearly presented. As is well-known, the celebrated theorem of Bombieri-Vinogradov has had an immense number of applications in analytic number theory and we foresee a similar role for our theorem. The desirable goal of course would be to have our theorem with the value $\eta=2$ but this seems to be far into the future.

\subsection{Outline of Proof}

Let $K$ be a number field with $n_{K}=[K: \mathbb{Q}]$, ring of integers $\mathcal{O}_{K}$, and let $d_{K}$ be the absolute value of the discriminant of $K$. Chebyshev's $\psi$ function for the number field $K$ is, in its simplest incarnation

$$
\psi(x)=\sum_{N \mathfrak{p}^{m} \leq x} \log N \mathfrak{p}
$$

where the sum is over all powers of unramified prime ideals $\mathfrak{p}$ of $K$ which satisfy the bound $N \mathfrak{p}^{m} \leq x$ where $N=N_{K / \mathbb{Q}}$. It is well known that an estimate of $\psi(x)$ can be translated to an estimate of $\pi(x)$, the number of prime ideals in $K$ of norm at most $x$. We will indicate the inclusion of ramified primes in a summation by the decoration $\sim$ over the function. We write

$$
\tilde{\psi}(x)=\sum_{N \mathfrak{c} \leq x} \Lambda(\mathfrak{c})
$$

where $\Lambda(\mathfrak{c})$ is the von Mangoldt function. That is,

$$
\Lambda(\mathfrak{c})=\left\{\begin{array}{cl}
\log N \mathfrak{p} & \text { if } \mathfrak{c} \text { is a power of the prime ideal } \mathfrak{p} \\
0 & \text { otherwise. }
\end{array}\right.
$$

We write $\psi(x, q, a)$ for the sum $\psi(x)$ restricted to those $\mathfrak{p}^{m}$ which satisfy $N \mathfrak{p}^{m} \equiv a(\bmod q)$. For ease of exposition, we will omit possible conjugacy class restrictions in this overview. Precise definitions will be given in subsequent sections. In particular, $\S 0.6$ contains definitions of the various Chebyshev functions. The aim of this paper is to prove a statement of the form

$$
\sum_{q \leq Q}^{\prime} \max _{(a, q)=1} \max _{y \leq x}\left|\psi(y, q, a)-\frac{y}{\phi(q)}\right| \ll \frac{x}{(\log x)^{A}}
$$

which is equivalent to the statement in the introduction.

To refine the summation, we rewrite the average as a sum over twisted Chebyshev functions, $\psi(x, \chi)$. In the simplest manifestation, $\chi$ is a Hecke character. We reserve the notation $\chi_{0}$ for the trivial character. The twisted Chebyshev function is

$$
\tilde{\psi}(x, \chi)=\sum_{N \mathfrak{c} \leq x} \Lambda(\mathfrak{c}) \chi(\mathfrak{c})
$$


and is the partial coefficient sum of the logarithmic derivative, $-\frac{L^{\prime}}{L}(s, \chi)$, of the $L$-series

$$
L(s, \chi)=\sum_{\mathfrak{c} \subset \mathcal{O}_{K}} \frac{\chi(\mathfrak{c})}{N \mathfrak{c}^{s}} .
$$

This connection with $L$-functions will be used extensively. We first convert the average on the left hand side of $(*)$ to one of the form

$$
\frac{1}{Q} \sum_{q \leq Q} \sum_{\chi}^{*} \max _{y \leq x}\left|\psi_{k}(y, \chi)\right|
$$

where the decoration $*$ indicates that the summation is over primitive characters only. That is, to prove $(*)$ we show that it is sufficient to prove that this new summation is $O\left(x(\log x)^{-A}\right)$. We then reinterpret this summation so that we can reduce to the case where the associated $L$-functions are all abelian, and estimate this new summation. We break the estimate into two cases, depending on the size of $Q$. We estimate the initial range

$$
Q \leq(\log x)^{\gamma}
$$

for any positive constant $\gamma$ using classical techniques in $\S 2$. In $\S 3$ we estimate the terminal range, for $Q$ in the range

$$
(\log x)^{\gamma} \leq Q \leq \min \left\{x^{\frac{1}{2}-\epsilon}, x^{\frac{1}{d-2}-\epsilon}\right\}
$$

(with $d=n_{E}$ for $E \subset K$ as described in Proposition 1.8 and $\gamma$ defined in Proposition 3.1) using the large sieve. To do so we will smooth the Chebyshev functions with an inverse Mellin transform. Specifically, for $f: \mathbb{R}_{\geq 1} \rightarrow \mathbb{R}$ define $f_{0}=f$ and for $k \in \mathbb{N}$ recursively define the inverse Mellin transforms $f_{k}$ of $f$ as

$$
f_{k}(x)=\int_{1}^{x} f_{k-1}(t) \frac{d t}{t} .
$$

We will use the notation $\exp (x)=e^{x}$.

\subsection{Class Functions}

If $f: G \rightarrow \mathbb{C}$ is a $\mathbb{C}$-valued function on a group $G$ and $\sigma \in G$ we define $f^{\sigma}: G \rightarrow \mathbb{C}$ by $f^{\sigma}(g)=f\left(\sigma^{-1} g \sigma\right)$. We say that $f$ is a class function if $f^{\sigma}=f$ for all $\sigma \in G$. We use class functions to impose conditions on the primes in our summation using the Frobenius, which we now define.

Let $L / M$ be Galois with group $G$ and $N=N_{M / \mathbb{Q}}$. For each prime ideal $\mathfrak{p}$ of $M$ and prime ideal $\mathfrak{q}$ of $L$ lying over $\mathfrak{p}$ the decomposition group, $D_{\mathfrak{q}}$, is the stabilizer of $\mathfrak{q}$ in $G,\{g \in G: g(\mathfrak{q})=\mathfrak{q}\}$. The decomposition group is isomorphic to $\operatorname{Gal}\left(L_{\mathfrak{q}} / M_{\mathfrak{p}}\right)$ where $L_{\mathfrak{q}}\left(\operatorname{resp} . M_{\mathfrak{p}}\right)$ is the completion of $L(\operatorname{resp} . M)$ at $\mathfrak{q}($ resp. $\mathfrak{p})$. Let $m_{\mathfrak{p}}$ denote the residue field $\mathcal{O}_{M} / \mathfrak{p}$ and $\ell_{\mathfrak{q}}$ the residue field $\mathcal{O}_{L} / \mathfrak{q}$. By Hensel's lemma there is a surjective map from $D_{\mathfrak{q}}$ to $\operatorname{Gal}\left(\ell_{\mathfrak{q}} / m_{\mathfrak{p}}\right)$, giving the exact sequence

$$
1 \rightarrow I_{\mathfrak{q}} \rightarrow D_{\mathfrak{q}} \rightarrow \operatorname{Gal}\left(\ell_{\mathfrak{q}} / m_{\mathfrak{p}}\right) \rightarrow 1
$$

where $I_{\mathfrak{q}}$ is the kernel of this map. The group $\operatorname{Gal}\left(\ell_{\mathfrak{q}} / m_{\mathfrak{p}}\right)$ is cyclic with generator $x \mapsto x^{N \mathfrak{p}}$ and $N \mathfrak{p}$ is the cardinality of $m_{\mathfrak{p}}$. The element of $D_{\mathfrak{q}}$ whose image in $\operatorname{Gal}\left(\ell_{\mathfrak{q}} / m_{\mathfrak{p}}\right)$ is a generator is well defined modulo $I_{\mathfrak{q}}$. Denote this element of $D_{\mathfrak{q}} / I_{\mathfrak{q}}$ by $\sigma_{\mathfrak{q}}$. If $\mathfrak{p}$ is unramified in $L$, then $I_{\mathfrak{q}}=1$ and we can consider $\sigma_{\mathfrak{q}}$ as an element of $G$, the Frobenius element. This element is characterized by

$$
\sigma_{\mathfrak{q}}(x)=x^{N \mathfrak{p}} \quad(\bmod \mathfrak{q})
$$

for all $x \in L$. For another choice $\mathfrak{q}^{\prime}$ above $\mathfrak{p}, I_{\mathfrak{q}^{\prime}}$ and $D_{\mathfrak{q}^{\prime}}$ are conjugates of $I_{\mathfrak{q}}$ and $D_{\mathfrak{q}}$ respectively in $G$. We let $\sigma_{\mathfrak{p}}$ denote this conjugacy class in $G$.

If $\mathfrak{p}$ is ramified, and $f$ is a class function then we define $f\left(\sigma_{\mathfrak{p}}^{m}\right)$ by extending $f$ to powers of the ramified primes by setting the value to be the average

$$
f\left(\sigma_{\mathfrak{p}}^{m}\right)=\frac{1}{\left|I_{\mathfrak{q}}\right|} \sum f(g)
$$


where the sum is over all $g \in D_{\mathfrak{q}}$ whose image in $D_{\mathfrak{q}} / I_{\mathfrak{q}}$ is $\sigma_{\mathfrak{q}}^{m}$.

For a subgroup $H$ of $G$, and a class function $f: H \rightarrow \mathbb{C}$ we define the induced class function

$$
\operatorname{Ind}_{H}^{G} f: G \rightarrow \mathbb{C}
$$

as follows. First, we extend $f$ to a function on $G$ by setting $f$ to be trivial outside of $H$. Let $g_{1}, \ldots, g_{R}$ be coset representatives for $H$ in $G$. We define

$$
\left(\operatorname{Ind}_{H}^{G} f\right)(g)=\sum_{r=1}^{R} f^{g_{r}}(g)=\frac{1}{|H|} \sum_{s \in G} f^{s}(g) .
$$

For a finite group $G$ and $f_{1}, f_{2}: G \rightarrow \mathbb{C}$ two $\mathbb{C}$-valued functions on $G$, we define their inner product

$$
\left\langle f_{1}, f_{2}\right\rangle=\frac{1}{|G|} \sum_{g \in G} f_{1}(g) \overline{f_{2}}(g) .
$$

\section{$0.3 \quad$ Artin $L$-series}

Our references for Artin $L$-series are [15] and [6]. Let $L / M$ be Galois with group $G$. (We suppress the field extension in the notation unless there is need for clarification.) Consider a representation, $\rho: G \rightarrow \mathrm{GL}_{n}(\mathbb{C})$ with character $\chi$. For $\operatorname{Re}(s)>1$ we define the unramified $L$-series associated to $\rho$ (or $\chi$ ), $L_{u}(s, \chi)$, to be a product of local Euler factors, where the product is taken over the primes ideals of $M$ unramified in $L$. Specifically,

$$
L_{u}(s, \chi)=\prod_{\mathfrak{p}}^{\prime} L_{\mathfrak{p}}(s, \chi)
$$

where the decoration ' indicates that the product is over those $\mathfrak{p}$ in $M$ which are unramified in $L$. The Euler factors are

$$
L_{\mathfrak{p}}(s, \chi)=\operatorname{det}\left(I-\rho\left(\sigma_{\mathfrak{q}}\right) N \mathfrak{p}^{-s}\right)^{-1}
$$

where $N=N_{M / \mathbb{Q}}$. We extend this $L$-series to ramified primes as follows. Let $\mathfrak{p}$ be a prime ideal of $M$ ramified in $L$ and $\mathfrak{q}$ a prime ideal in $L$ above $\mathfrak{p}$. Let $V$ be the underlying complex vector space on which $\rho$ acts. Restricting this action to the decomposition group $D_{\mathfrak{q}}$, the quotient $D_{\mathfrak{q}} / I_{\mathfrak{q}}$ acts on the subspace $V^{I_{\mathfrak{q}}}$ of $V$ on which $I_{\mathfrak{q}}$ acts trivially. All $\sigma_{\mathfrak{q}}$ have identical characteristic polynomials on this subspace, and we define the Euler product at the ramified prime ideals $\mathfrak{p}$ to be

$$
L_{\mathfrak{p}}(s, \chi)=\operatorname{det}\left(I-\left.\rho\left(\sigma_{\mathfrak{q}}\right)\right|_{V^{I_{\mathfrak{q}}}} N \mathfrak{p}^{-s}\right)^{-1} .
$$

For all primes ideals $\mathfrak{p}$, each factor $L_{\mathfrak{p}}$ depends only on $\mathfrak{p}$ and has degree $\chi(1)$, the dimension of $\rho$. For each factor $L_{\mathfrak{p}}(s, \chi)$,

$$
\log L_{\mathfrak{p}}(s, \chi)=\sum_{m=1}^{\infty} \chi\left(\sigma_{\mathfrak{p}}^{m}\right) m^{-1} N \mathfrak{p}^{-m s}
$$

where $\chi\left(\sigma_{\mathfrak{p}}^{m}\right)$ is the trace of $\rho\left(\sigma_{\mathfrak{p}}^{m}\right)$ if $\mathfrak{p}$ is unramified in $L$, and we extend $\chi$ to the ramified prime ideals as discussed in $\S 0.2$. The (full) Artin $L$-series is

$$
L(s, \chi)=\prod_{\mathfrak{p}} L_{\mathfrak{p}}(s, \chi)
$$

where the product is over all primes ideals $\mathfrak{p}$ in $M$. We can rewrite this product as

$$
L(s, \chi)=\prod_{p} L_{p}(s, \chi)
$$


where this product is over rational primes $p$, and $L_{p}(s, \chi)=\prod_{\mathfrak{p} \mid p} L_{\mathfrak{p}}(s, \chi)$. Each $L_{p}$ is a polynomial in $p^{-s}$ of degree, say $m_{p}$. Note that $m_{p} \leq n_{M}$ for all $p$ and $m_{p}=n_{M}$ for $p$ unramified in $M$. The Euler factors can be written as

$$
L_{p}(s, \chi)=\prod_{j=1}^{m_{p}}\left(1-\frac{\pi_{p, j}}{p^{s}}\right)^{-1}
$$

where $\left|\pi_{p, j}\right|=1$. From the logarithms of the Euler factors it is apparent that the logarithmic derivative of $L(s, \chi)$ is

$$
\frac{L^{\prime}}{L}(s, \chi)=-\sum_{\mathfrak{p}} \sum_{m=1}^{\infty}(\log N \mathfrak{p}) \chi\left(\sigma_{\mathfrak{p}}^{m}\right) N \mathfrak{p}^{-m s} .
$$

With the induced function defined in $\S 0.2$, if $H$ is a subgroup of $G$ and $L^{H}$ is the subfield of $L$ fixed by $H$ then for a character $\chi$ of $H$

$$
L\left(s, \operatorname{Ind}_{H}^{G} \chi, L / M\right)=L\left(s, \chi, L / L^{H}\right) .
$$

\subsection{Functional Equations}

Our reference for this material is [15]. For $L / M$ Galois we now define an enlarged $L$-function, $\Lambda$, from which a functional equation will follow. First, we define the gamma factors. Let $\gamma(s)=\pi^{-\frac{s}{2}} \Gamma\left(\frac{s}{2}\right)$. For each infinite complex place $\nu$ of $M$, define the local factor $\gamma_{\nu}(s, \chi)=[\gamma(s) \gamma(s+1)]^{\chi(1)}$. Now assume that $\nu$ is real. To a place $\omega$ of $L$ above $\nu$ there corresponds a decomposition group $G(\omega)=\{g \in G: g(\omega)=\omega\}$ which is of order one or two. The generator $\sigma_{\omega}$ is well defined up to conjugation by $\nu$. Let $V$ be the underlying representation space for $\rho$. The vector space $V$ decomposes as a direct sum, $V=V_{\nu}^{+} \oplus V_{\nu}^{-}$corresponding to the +1 and -1 eigenvalues of $\rho\left(\sigma_{\omega}\right)$. We define the local factor

$$
\gamma_{\nu}(s, \chi)=\gamma(s)^{\operatorname{dim} V_{\nu}^{+}} \gamma(s+1)^{\operatorname{dim} V_{\nu}^{-}}
$$

and define $\gamma(s, \chi)=\prod_{\nu} \gamma_{\nu}(s, \chi)$. We can write $\gamma(s, \chi)=\gamma(s)^{a(\chi)} \gamma(s+1)^{b(\chi)}$ for integers $a(\chi)+b(\chi)=$ $n_{M} \chi(1)$. Before we define $\Lambda$ we need to introduce the notion of a conductor.

The Artin conductor $f(\chi)$ associated to $\chi$ is an ideal of $\mathcal{O}_{M}$ defined as follows. Let $\mathfrak{p}$ be a prime ideal of $M$ and $\mathfrak{q}$ a prime ideal of $L$ lying over $\mathfrak{p}$. Let $G_{0}$ denote the inertia group $I_{\mathfrak{q}}$ at $\mathfrak{q}$. There is a descending filtration of higher ramification groups $G_{0} \supseteq G_{1} \supset \ldots$ The quantity

$$
n(\chi, \mathfrak{p})=\sum_{i=0}^{\infty} \frac{\left|G_{i}\right|}{\left|G_{0}\right|} \operatorname{codim}\left(V^{G_{i}}\right)
$$

is an integer which is independent of the choice of $\mathfrak{q}$ above $\mathfrak{p}$. It is equal to zero for all prime ideals $\mathfrak{p}$ unramified in $L$, and therefore vanishes for all but finitely many $\mathfrak{p}$. The Artin conductor is the ideal $\mathfrak{f}(\chi)=\prod_{\mathfrak{p}} \mathfrak{p}^{n(\chi, \mathfrak{p})}$ and the conductor is the constant

$$
A(\chi)=d_{M}^{\chi(1)} N_{M / \mathbb{Q}} \mathfrak{f}(\chi) .
$$

If $\chi$ is an Artin character induced by a Dirichlet character of modulus $q$, with $A=A\left(\chi_{0}\right)$ it follows that $A(\chi) \ll A q^{n_{M}}$. In this case, $\chi(1)=1$ and the Artin conductor is $q$, so $A(\chi) \leq d_{M} N_{M / \mathbb{Q}} q \leq d_{M} q^{n_{M}} \ll q^{n_{M}}$. If $L=K\left(\zeta_{q}\right), \chi_{1}$ is a character induced by $\operatorname{Gal}(K / \mathbb{Q})$, and $\chi_{2}$ is induced by a Dirichlet character modulo $q$, then in this case $A\left(\chi_{1} \otimes \chi_{2}\right)=A\left(\chi_{1}\right) A\left(\chi_{2}\right)$. Therefore, $A\left(\chi_{1} \otimes \chi_{2}\right) \ll A\left(\chi_{1}\right) q^{n_{M}}$. A simple case of the conductor discriminant formula is the relation

$$
d_{L}=d_{M}^{[L: M]} N_{M / \mathbb{Q}}\left(d_{L / M}\right)
$$

where $d_{L / M}$ is the relative discriminant,

$$
d_{L / M}=\prod_{\chi} \mathfrak{f}(\chi)^{\chi(1)}
$$

and the product is over all irreducible characters $\chi$ of $\operatorname{Gal}(L / M)$. (See [19] Proposition 4.8.) 
We now define $\Lambda(s, \chi)=A(\chi)^{\frac{s}{2}} \gamma(s, \chi) L(s, \chi)$ for $\operatorname{Re}(s)>1$, which has a meromorphic continuation to the whole complex plane and functional equation

$$
\Lambda(s, \chi)=W(\chi) \Lambda(1-s, \bar{\chi})
$$

where $W(\chi)$, the Artin number, is a complex number of absolute value 1 . The character $\bar{\chi}$ is the complex conjugate of $\chi$. That is, if $\chi$ is the character of the representation $\rho: G \rightarrow \operatorname{GL}(V)$, then $\bar{\chi}$ is the character of the contragradient representation $\bar{\rho}: G \rightarrow \mathrm{GL}\left(V^{*}\right)$ where $V^{*}$ is the dual of $V$ and $\bar{\rho}$ is defined by

$$
\left\langle\bar{\rho}_{g}(f), v^{*}\right\rangle=\left\langle f, \rho_{g}^{-1}(v)\right\rangle
$$

for all $g \in G, v \in V$ and $v^{*} \in V^{*}$.

It will be convenient to set

$$
\Theta(s, \chi)=W(\chi) A(\chi)^{\frac{1}{2}-s} \gamma(1-s, \bar{\chi}) \gamma(s, \chi)^{-1} .
$$

With this notation we can rewrite the functional equation as

$$
L(s, \chi)=\Theta(s, \chi) L(1-s, \bar{\chi})
$$

since $A(\chi)=A(\bar{\chi})$.

We will use Stirling's inequality in various forms. For $\sigma$ fixed, as $|t| \rightarrow \infty$, (see [16] ex. 6.3.15)

$$
\Gamma(\sigma+i t) \sim e^{-\frac{1}{2} \pi|t|}|t|^{\sigma-\frac{1}{2}} \sqrt{2 \pi} .
$$

For $s=\sigma+i t$,

$$
\Theta(s, \chi) \ll\left[A(\chi)(|t|+2)^{n_{M}}\right]^{\frac{1}{2}-\sigma} .
$$

Logarithmically differentiating (0.4.2) and applying Stirling's formula, we also have

$$
\frac{\Theta^{\prime}}{\Theta}(s, \chi) \ll n_{M}\left(\log (|t|+2)+\frac{1}{|s|}\right) .
$$

\subsection{Congruences and Characteristic Functions}

Let $K / M$ be Galois with group $G$. In the average, we wish to estimate the number of prime ideals $\mathfrak{p}$ in $M$ which are unramified in $K$ such that $\sigma_{\mathfrak{p}}=C$ for a fixed conjugacy class $C$ of $G$. We extend class functions to the ramified primes as in $\S 0.2$. Let $\delta_{C}: G \rightarrow\{0,1\}$ denote the characteristic function of $C$. By the orthogonality relations of characters, for a fixed element $g$ of $C$,

$$
\delta_{C}=\frac{|C|}{|G|} \sum_{\eta} \bar{\eta}(g) \eta
$$

where the sum is over irreducible characters $\eta$ of $G$. An alternate decomposition is as follows. Let $H$ be a subgroup of $G$ such that $H \cap C \neq \emptyset$. Let $h \in H \cap C$ and let $C_{H}$ be the conjugacy class of $h$ in $H$. With $\lambda=\frac{|C||H|}{|G|\left|C_{H}\right|}$, and the induced character as defined in $\S 0.2$,

$$
\delta_{C}=\lambda \operatorname{Ind}_{H}^{G} \delta_{C_{H}} .
$$

We wish to count only those prime powers $\mathfrak{p}^{m}$ so that $N_{M / \mathbb{Q}} \mathfrak{p}^{m} \equiv a(\bmod q)$, and $\sigma_{\mathfrak{p}}^{m}=C$. If $K \cap \mathbb{Q}\left(\zeta_{q}\right)=$ $\mathbb{Q}$, with $G_{q}=\operatorname{Gal}\left(K\left(\zeta_{q}\right) / M\right)$,

$$
G_{q} \cong G \times \operatorname{Gal}\left(\mathbb{Q}\left(\zeta_{q}\right) / \mathbb{Q}\right) \cong G \times(\mathbb{Z} / q \mathbb{Z})^{\times} .
$$

Fixing this isomorphism, let the conjugacy class $C^{\prime}$ be the class associated to $\left(C, C_{2}\right)$ where $C$ is the preferred class in $G$ and $C_{2}$ is a class in $(\mathbb{Z} / q \mathbb{Z})^{\times}$corresponding to the congruence condition. Let $\delta_{C}$ and $\delta_{a, q}=\delta_{C_{2}}$ be characteristic functions on $G_{q}$. (For an element $(g, t) \in G_{q}$, define $\delta_{C}(g, t)=1$ if $g \in C$ and 0 otherwise. The function $\delta_{a, q}$ is defined in a similar manner.) 
Definition 0.1. Let $\xi=\xi(C, a, q)=\delta_{C^{\prime}}=\delta_{C} \otimes \delta_{a, q}$.

For a subgroup $H$ of $G$ with $H \cap C \neq \emptyset$ set $H_{q}=H \times \operatorname{Gal}\left(\mathbb{Q}\left(\zeta_{q}\right) / \mathbb{Q}\right)$. If $\chi$ is a Dirichlet character of modulus $q$, then $\chi$ induces an Artin character by defining $\chi\left(\sigma_{p}^{m}\right)=\chi\left(N \mathfrak{p}^{m}\right)$ since as $M \cap \mathbb{Q}\left(\zeta_{q}\right)=\mathbb{Q}$ in the extension $M\left(\zeta_{q}\right) / M$, the conjugacy class of $N \mathfrak{p}$ determines the Frobenius element. By Mackey's induction theorem,

$$
\delta_{C} \otimes \chi=\lambda \operatorname{Ind}_{H_{q}}^{G_{q}}\left(\delta_{C_{H}} \otimes \chi\right)
$$

where we have used $\chi$ for the character on both $G_{q}$ and $H_{q}$ induced by the character $\chi$ modulo $q$.

As in (0.3.3),

$$
L\left(s, \delta_{C} \otimes \chi, L / M\right)=L\left(s, \operatorname{Ind}_{H_{q}}^{G_{q}}\left(\delta_{C_{H}} \otimes \chi\right), L / M\right)^{\lambda}=L\left(s, \delta_{C_{H}} \otimes \chi, L / L^{H}\right)^{\lambda}
$$

where $L^{H}$ is the fixed field of $H$.

\subsection{Chebyshev $\psi$ functions}

For $L / M$ Galois and a class function $f$ of $\operatorname{Gal}(L / M)$ we define

$$
\psi(x, f, L / M)=\sum_{N \mathfrak{p}^{m} \leq x}(\log N \mathfrak{p}) f\left(\sigma_{\mathfrak{p}}^{m}\right)
$$

where the sum is over powers of those prime ideals $\mathfrak{p}$ of $M$ unramified in $L$, and $N=N_{M / \mathbb{Q}}$. We suppress the field extension unless there is need for clarification. When $f$ is trivial this is $\psi(x)$. After smoothing with the inverse Mellin transform (0.1.1)

$$
\psi_{k}(x, f)=\frac{1}{k !} \sum_{N \mathfrak{p}^{m} \leq x}(\log N \mathfrak{p})\left(\log \frac{x}{N \mathfrak{p}^{m}}\right)^{k} f\left(\sigma_{\mathfrak{p}}^{m}\right) .
$$

To make the connection with $L$-functions precise, we include ramified primes in our summation. We do so by extending the class function $f$ to the ramified primes as in $\S 0.2$. We denote the inclusion of ramified primes by the decoration $\sim$ over the function.

For an Artin character $\chi$, if $\operatorname{Re}(s)>1$, by examining the logarithmic derivative of $L(s, \chi)$ (see $(0.3 .2)$ ) $\tilde{\psi}(x, \chi)$ is the partial sum of the coefficients, and

$$
\tilde{\psi}_{k}(x, \chi)=-\frac{1}{2 \pi i} \int_{(2)} \frac{L^{\prime}}{L}(s, \chi) \frac{x^{s}}{s^{k+1}} d s
$$

where the integration is on the line $\operatorname{Re}(s)=2$.

We will begin estimating an average involving the error term $\psi(x, C, q, a)-d(C, q, a) x$, and show that it is equivalent to one which includes ramified primes, corresponding to the function $\tilde{\psi}(x, C, q, a)=\tilde{\psi}\left(x, \xi, K\left(\zeta_{q}\right) / M\right)$, where $\xi$ is as in Definition 0.1. In turn, we will rewrite this average as one with functions of the form

$$
\tilde{\psi}\left(x, \delta_{C} \otimes \chi, K\left(\zeta_{q}\right) / M\right)=\sum_{N \mathfrak{p}^{m} \leq x}(\log N \mathfrak{p}) \delta_{C}\left(\sigma_{\mathfrak{p}}^{m}\right) \chi\left(N \mathfrak{p}^{m}\right)
$$

where $\chi$ is induced by a Dirichlet character of modulus $q$ and the sum is over powers of prime ideals $\mathfrak{p}$ in $M$. These functions can be smoothed by the inverse Mellin transform. Taking coefficient sums of (0.5.2) with the notation from $\S 0.5$

$$
\tilde{\psi}_{k}\left(x, \delta_{C} \otimes \chi, L / M\right)=\tilde{\psi}_{k}\left(x, \operatorname{Ind}_{H_{q}}^{G_{q}}\left(\delta_{C_{H}} \otimes \chi\right), L / M\right)^{\lambda}=\tilde{\psi}_{k}\left(x, \delta_{C_{H}} \otimes \chi, L / L^{H}\right)^{\lambda} .
$$

Writing the characteristic function $\xi=\frac{|C|}{|G|} \sum_{\eta} \bar{\eta}(g) \eta$ (see $\S 0.5$ ), for $\operatorname{Re}(s)>1$, using $(0.3 .2)$

$$
-\frac{L^{\prime}}{L}(s, \xi)=-\frac{|C|}{|G|} \sum_{\eta} \bar{\eta}(g) \frac{L^{\prime}}{L}(s, \eta)
$$


By $(0.6 .2)$

$$
\tilde{\psi}_{k}(x, \xi, L / M)=-\frac{1}{2 \pi i} \int_{(2)} \frac{L^{\prime}}{L}(s, \xi) \frac{x^{s}}{s^{k+1}} d s=-\frac{1}{2 \pi i} \frac{|C|}{|G|} \sum_{\eta} \bar{\eta}(g) \int_{(2)} \frac{L^{\prime}}{L}(s, \eta) \frac{x^{s}}{s^{k+1}} d s
$$

where the integration is on the line $\operatorname{Re}(s)=2$.

\subsection{Abelian $L$-functions}

In our previous definitions, the Artin characters are, in general, non-abelian characters of $\operatorname{Gal}(L / M)$. We now rewrite the $L$-functions in terms of abelian $L$-functions. We follow [13], using the methods of Deuring [8] and MacCluer [14]. In (0.6.4) we rewrote $\xi$ as a sum over irreducible characters using a preferred element $g$ of $G=\operatorname{Gal}(K / M)$. Let $H=\langle g\rangle$, the cyclic group generated by $g$ and let $E$ be the fixed field of $H$. Since $H$ is cyclic, the irreducible characters of $H$ are one-dimensional.

From (0.6.3)

$$
\tilde{\psi}\left(x, \delta_{C} \otimes \chi, L / M\right)=\lambda \tilde{\psi}\left(x, \delta_{C_{H}} \otimes \chi, L / E\right)
$$

where the characters on the right are one-dimensional by construction. We can further write the character $\delta_{C_{H}}$ as the sum over irreducible characters from (0.5.1)

$$
\delta_{C_{H}}=\frac{\left|C_{H}\right|}{|H|} \sum_{\eta} \bar{\eta}(g) \eta
$$

with $g \in H \cap C$. We conclude that

$$
\tilde{\psi}_{k}\left(x, \delta_{C} \otimes \chi, L / M\right)=\lambda \tilde{\psi}_{k}\left(x, \delta_{C_{H}} \otimes \chi, L / E\right)=\frac{|C|}{|G|} \sum_{\eta} \bar{\eta}(g) \tilde{\psi}_{k}(x, \eta \otimes \chi, L / E) .
$$

By $(0.6 .4)$

$$
\tilde{\psi}_{k}\left(x, \delta_{C} \otimes \chi, L / M\right)=-\frac{1}{2 \pi i} \frac{|C|}{|G|} \sum_{\eta} \bar{\eta}(g) \int_{(2)} \frac{L^{\prime}}{L}(s, \eta \otimes \chi, L / E) \frac{x^{s}}{s^{k+1}} d s .
$$

The motivation behind rewriting our functions in terms of abelian characters is the following, which are known to hold only in the abelian case. In the following, $L(s, \eta)$ is an abelian $L$-function and $\eta$ is assumed not to be the trivial character. (See [13] 432-434.) The Hadamard factorization, for constants $B_{1}(\eta)$ and $B(\eta)$ is

$$
\Lambda(s, \eta)=e^{B_{1}(\eta)+B(\eta) s} \prod_{\rho}\left(1-\frac{s}{\rho}\right) e^{\frac{s}{\rho}}
$$

where $\rho$ runs through all of the zeros of $\Lambda(s, \eta)$. These are the non-trivial zeros of $L(s, \eta)$, those for which $0<\operatorname{Re}(\rho)<1$. By logarithmic differentiation, using the above,

$$
\frac{L^{\prime}}{L}(s, \eta)=B(\eta)+\sum_{\rho}\left(\frac{1}{s-\rho}+\frac{1}{\rho}\right)-\frac{1}{2} \log A(\eta)-\frac{\gamma^{\prime}}{\gamma}(s, \eta)
$$

From [13] Lemma 5.1, $\operatorname{Re}(B(\eta))=-\sum_{\rho} \operatorname{Re}\left(\frac{1}{\rho}\right)$. Therefore,

$$
\frac{L^{\prime}}{L}(s, \eta)+\frac{L^{\prime}}{L}(s, \bar{\eta})=\sum_{\rho}\left(\frac{1}{s-\rho}+\frac{1}{s-\bar{\rho}}\right)-\frac{1}{2} \log A(\eta)-\frac{\gamma^{\prime}}{\gamma}(s, \eta)
$$

where the sum runs through all non-trivial zeros $\rho$ of $L(s, \eta)$. 


\section{Rewriting the Average}

We continue to consider $K / M$ Galois with group $G$, and let $L=K\left(\zeta_{q}\right)$. If $1 \leq a<q$ and $(a, q)=1$ and $K \cap \mathbb{Q}\left(\zeta_{q}\right)=\mathbb{Q}$, then $L / M$ is also Galois with group isomorphic to $G_{q}=\operatorname{Gal}(K / M) \times(\mathbb{Z} / q \mathbb{Z})^{\times}$. We let $\xi=\xi(C, q, a)=\delta_{C} \otimes \delta_{a, q}$ be the class function from Definition 0.1. Therefore, $\tilde{\psi}(x, C, q, a)=\tilde{\psi}(x, \xi, L / M)$. We wish to estimate the average (for a suitable choice of $Q_{1}$ )

$$
\sum_{q \leq Q_{1}}^{\prime} \max _{(a, q)=1} \max _{y \leq x}|\psi(y, C, q, a)-d(C, q, a) y|
$$

where the decoration ' on the summation indicates that the sum is over those $q$ so that $K \cap \mathbb{Q}\left(\zeta_{q}\right)=\mathbb{Q}$. For these $q, d(C, q, a)=\frac{|C|}{|G|} \frac{1}{\phi(q)}$. To simplify expressions, we will let $r_{k}(x, C, q, a)=\psi_{k}(x, C, q, a)-d(C, q, a) x$ and decorate $r$ to mirror the corresponding $\psi$ function. The goal of this section is to prove Proposition 1.8, which states that in order to show that the above average is $O\left(x(\log x)^{-A}\right)$ it suffices to show that for all values $Q \leq Q_{1}$ there is a $k \geq 0$ so that the average

$$
\frac{1}{Q} \sum_{q \leq Q}^{\prime} \sum_{\chi}^{*} \max _{y \leq x}\left|\tilde{\psi}_{k}\left(y, \chi, K\left(\zeta_{q}\right) / E\right)\right| \ll \frac{x}{(\log x)^{A}}
$$

where $K / E$ is abelian.

First, we show that the contribution of the ramified primes is negligible.

Lemma 1.1. Assume that $1 \leq a<q$ and $(a, q)=1$ with $K \cap \mathbb{Q}\left(\zeta_{q}\right)=\mathbb{Q}$. Then with

$$
E_{1}=E_{1}(x, k)=\frac{1}{k !}(\log x)^{k+1}\left(\log d_{K}+n_{M} x^{\frac{1}{2}}\right)
$$

the following hold.

1. $\psi_{k}(x, C, q, a)-\tilde{\psi}_{k}(x, \xi) \ll E_{1}$.

2. $\sum_{q \leq Q_{1}} \max _{(a, q)=1} \max _{y \leq x}\left|r_{k}(y, C, q, a)\right|-\sum_{q \leq Q_{1}} \max _{(a, q)=1} \max _{y \leq x}\left|\tilde{r}_{k}(y, \xi)\right| \ll Q_{1} E_{1}$.

Proof. First we prove 1. The number of prime ideals $\mathfrak{p}$ of $M$ ramified in $K$ is at most

$$
\sum_{\mathfrak{p} \mid d_{K / M}} \frac{\log N \mathfrak{p}}{\log 2} \leq \frac{\log N\left(d_{K / M}\right)}{\log 2},
$$

with $N=N_{M / \mathbb{Q}}$ and $d_{K / M}$ the relative discriminant of $K / M$. By the conductor discriminant formula (0.4.1), $d_{K}=d_{M}^{[K: M]} N\left(d_{K / M}\right)$. We conclude that the number of ramified primes is at most $\log d_{K}$.

Using definition (0.6.1) the difference in part 1 is the following sum over ramified prime powers

$$
\begin{aligned}
\frac{1}{k !} \sum_{N \mathfrak{p}^{m} \leq x}(\log N \mathfrak{p})\left(\log \frac{x}{N \mathfrak{p}}\right)^{k} \xi\left(\sigma_{\mathfrak{p}}^{m}\right) & \leq \frac{1}{k !}(\log x)^{k+1}\|\xi\|\left(\sum_{N \mathfrak{p} \leq x} 1+\sum_{\substack{N \mathfrak{p}^{m} \leq x \\
m>1}} 1\right) \\
& \leq \frac{1}{k !}(\log x)^{k+1}\left(\log d_{K}+n_{M} x^{\frac{1}{2}}\right),
\end{aligned}
$$

where $\|\xi\|=\max _{\mathfrak{p}, m}\left|\xi\left(\sigma_{\mathfrak{p}}^{m}\right)\right| \leq 1$ since $\xi$ is a characteristic function. This proves part 1 .

For part 2, from 1, the triangle inequality implies that $\left|\psi_{k}(y, C, q, a)-d(C, q, a) y\right|-\left|\tilde{\psi}_{k}(y, \xi)-d(C, q, a) y\right|=$ $\left|r_{k}(y, C, q, a)\right|-\left|\tilde{r}_{k}(y, \xi)\right| \ll E_{1}$. Hence, it suffices to estimate

$$
\sum_{q \leq Q_{1}} \max _{(a, q)=1} \max _{y \leq x} E_{1}(y, k) \ll Q_{1} E_{1}(x, k) .
$$


We now show that the difference between iterations of the Mellin transform, even incorporating ramified primes is negligible.

Lemma 1.2. Assume that $1 \leq a<q$ and $(a, q)=1$ with $K \cap \mathbb{Q}\left(\zeta_{q}\right)=\mathbb{Q}$. Let $A>1$ and a positive integer $n$ be given. Then with

$$
E_{2}=E_{2}\left(x, k, n, A, Q_{1}\right)=\frac{1}{(k+n) !}(\log x)^{\frac{1}{2} n(n+1) A+k+n+1} Q_{1}\left(\log Q_{1}+n_{M} x^{\frac{1}{2}}\right)+\frac{x|C|\left(\log Q_{1}\right)\left(\log \log Q_{1}\right)}{|G|(\log x)^{A}}
$$

the following hold.

$$
\begin{aligned}
& \text { 1. } r_{k}(x, C, q, a) \ll \frac{d(C, q, a) x}{(\log x)^{A}}+(\log x)^{\frac{1}{2} n(n+1) A} \max _{y \leq x e^{n(\log x)-A}}\left|r_{k+1}(y, C, q, a)\right| . \\
& \text { 2. } \sum_{q \leq Q_{1}}^{\prime} \max _{(a, q)=1} \max _{y \leq x}\left|r_{k}(y, C, q, a)\right| \ll(\log x)^{\frac{1}{2} n(n+1) A} \sum_{q \leq Q_{1}}^{\prime} \max _{(a, q)=1} \max _{y \leq x e^{n(\log x)}-A}\left|\tilde{r}_{k+n}(y, \xi)\right|+E_{2} .
\end{aligned}
$$

Proof. Let $\psi_{k}^{*}(x)=\psi_{k}(x, C, q, a)$. For the first part of the proof we will let $d^{*}=d(C, q, a)$. Since $\psi_{k}^{*}$ is increasing, for any $0<\alpha \leq 1$, using the mean value thoerem one can show the following identity

$$
\frac{1}{\alpha} \int_{e^{-\alpha} x}^{x} \psi_{k}^{*}(t) \frac{d t}{t} \leq \psi_{k}^{*}(x) \leq \frac{1}{\alpha} \int_{x}^{e^{\alpha} x} \psi_{k}^{*}(t) \frac{d t}{t} .
$$

We can rewrite this as

$$
\frac{1}{\alpha}\left(\psi_{k+1}^{*}(x)-\psi_{k+1}^{*}\left(e^{-\alpha} x\right)\right) \leq \psi_{k}^{*}(x) \leq \frac{1}{\alpha}\left(\psi_{k+1}^{*}\left(e^{\alpha} x\right)-\psi_{k+1}^{*}(x)\right) .
$$

First, we will show

$$
r_{k}^{*}(x) \ll \alpha d^{*} x+\frac{1}{\alpha} \max _{y=x, x e^{ \pm \alpha}}\left|r_{k+1}^{*}(y)\right| .
$$

Substituting the remainder terms into $(*)$ we have

$$
\begin{aligned}
\alpha \psi_{k}^{*}(x) & \leq \psi_{k+1}^{*}\left(e^{\alpha} x\right)-\psi_{k+1}^{*}(x) \\
& =d^{*} e^{\alpha} x+r_{k+1}^{*}\left(e^{\alpha} x\right)-d^{*} x-r_{k+1}^{*}(x) \\
& =\left(r_{k+1}^{*}\left(e^{\alpha} x\right)-r_{k+1}^{*}(x)\right)+d^{*} x\left(e^{\alpha}-1\right) \\
& =\left(r_{k+1}^{*}\left(e^{\alpha} x\right)-r_{k+1}^{*}(x)\right)+\alpha d^{*} x+O\left(\alpha^{2} d^{*} x\right) .
\end{aligned}
$$

The last equality can be verified using the Taylor series expansion of $e^{\alpha}$. We conclude that $r_{k}^{*}(x) \leq$ $\frac{1}{\alpha}\left(r_{k+1}^{*}\left(e^{\alpha} x\right)-r_{k+1}^{*}(x)\right)+O\left(\alpha d^{*} x\right)$. Similarly, $r_{k}^{*}(x) \geq \frac{1}{\alpha}\left(r_{k+1}^{*}(x)-r_{k+1}^{*}\left(e^{\alpha} x\right)\right)+O\left(\alpha d^{*} x\right)$. Together these imply (3.1.1).

Iteratively applying (3.1.1) with $\alpha, \alpha^{2}, \ldots, \alpha^{n}$ we have $r_{k}^{*}(x) \ll \alpha_{1} d^{*} x+\alpha_{2} \max _{y \leq x e^{\alpha_{3}}}\left|r_{k+1}^{*}(y)\right|$ where

$$
\begin{aligned}
& \alpha_{1}=\alpha+\alpha e^{\alpha}+\alpha e^{\alpha+\alpha^{2}}+\cdots+\alpha e^{\alpha+\cdots+\alpha^{n-1}} \ll \alpha\left(n e^{n-1}\right) \ll \alpha \\
& \alpha_{2}=\alpha^{-\frac{1}{2} n(n+1)} \\
& \alpha_{3}=\alpha+\alpha^{2}+\cdots+\alpha^{n} \leq n \alpha .
\end{aligned}
$$

This implies that $r_{k}^{*}(x) \ll \alpha d^{*} x+\alpha^{-\frac{1}{2} n(n+1)} \max _{y \leq x e^{n \alpha}}\left|r_{k+n}^{*}(y)\right|$. Choosing $\alpha=(\log x)^{-A}$ completes the proof of 1 .

Summing 1 implies that

$$
\sum_{q \leq Q_{1}}^{\prime} \max _{(a, q)=1} \max _{y \leq x}\left|r_{k}^{*}(y)\right| \ll(\log x)^{\frac{1}{2} n(n+1) A} \sum_{q \leq Q_{1}}^{\prime} \max _{(a, q)=1} \max _{y \leq x e^{n(\log x)-A}}\left|r_{k+n}^{*}(y)\right|+\sum_{q \leq Q_{1}}^{\prime} \frac{d(C, q, a) x}{(\log x)^{A}} .
$$


Focusing on the error term, since

$$
\sum_{q \leq Q_{1}}^{\prime} d(C, q, a)=\frac{|C|}{|G|} \sum_{q \leq Q_{1}}^{\prime} \frac{1}{\phi(q)} \text { and } \sum_{q \leq Q_{1}} \frac{1}{\phi(q)} \ll\left(\log Q_{1}\right)\left(\log \log Q_{1}\right)
$$

(see $[11] \S 18.4$ ) we conclude that

$$
\sum_{q \leq Q_{1}}^{\prime} \frac{d(C, q, a) x}{(\log x)^{A}} \ll \frac{|C| x\left(\log Q_{1}\right)\left(\log \log Q_{1}\right)}{|G|(\log x)^{A}} .
$$

Therefore

$$
\sum_{q \leq Q_{1}}^{\prime} \max _{(a, q)=1} \max _{y \leq x}\left|r_{k}^{*}(y)\right| \ll(\log x)^{\frac{1}{2} n(n+1) A} \sum_{q \leq Q_{1}}^{\prime} \max _{(a, q)=1} \max _{y \leq x e^{n(\log x)^{-A}}}\left|r_{k+n}^{*}(y)\right|+\frac{|C| x\left(\log Q_{1}\right)\left(\log \log Q_{1}\right)}{|G|(\log x)^{A}} .
$$

With Lemma 1.1 part 2 this directly proves part 2 as $e^{(\log x)^{-A}}$ is bounded when $A>1$.

Using the following identity for Dirichlet characters $\chi$ modulo $q$,

$$
\sum_{\chi} \bar{\chi}(a) \chi(n)=\left\{\begin{array}{cl}
\phi(q) & \text { if } n \equiv a \quad(\bmod q) \\
0 & \text { otherwise }
\end{array}\right.
$$

it follows that

$$
\tilde{\psi}_{k}(x, \xi)=\frac{1}{\phi(q)} \sum_{\chi(q)} \bar{\chi}(a) \tilde{\psi}_{k}\left(x, \delta_{C} \otimes \chi\right) .
$$

The trivial character contributes the term $\tilde{\psi}_{k}\left(x, \delta_{C}\right)$ and

$$
\tilde{r}_{k}(x, \xi)=\tilde{\psi}_{k}(x, \xi)-d(C, q, a) x=\frac{1}{\phi(q)} \sum_{\chi \neq \chi_{0}} \bar{\chi}(a) \tilde{\psi}_{k}\left(x, \delta_{C} \otimes \chi\right)+\frac{1}{\phi(q)}\left(\tilde{\psi}_{k}\left(x, \delta_{C}\right)-\frac{|C|}{|G|} x\right) .
$$

We bound the second term on the right by the effective Chebotarev density theorem in the form of Lemma 1.5 part 1. The first term is estimated in Lemma 1.5 part 2. The Chebotarev density theorem we require is a combination of a slight modification of Theorem 1.3 of [13] and Theorem 1' of [22] (as mentioned in [13]). We state these results below.

Theorem $1.3\left([13]\right.$ Theorem 1.3). Assume $K \neq \mathbb{Q}$. There is at most one zero of $\zeta_{K}(s)$ in the region defined by $s=\sigma+i t$ with

$$
1-\left(4 \log d_{K}\right)^{-1} \leq \sigma \leq 1, \quad|t| \leq\left(4 \log d_{K}\right)^{-1} .
$$

If such a zero exists, it is real and simple. Let $\beta$ denote this zero if it exists. If $x \geq \exp \left(10 n_{K}\left(\log d_{K}\right)^{2}\right)$ then

$$
r\left(x, \delta_{C}\right) \ll E_{3}(x)=\frac{|C|}{|G|} x^{\beta}+x \exp \left(-c n_{K}^{-\frac{1}{2}}(\log x)^{\frac{1}{2}}\right),
$$

where the $\beta$ term in $E_{3}$ is present only if such an exceptional zero exists, and the implied constant is effective and absolute.

We will combine this with a result of Stark's to bound such a $\beta$. These two theorems give an effective bound on the error term.

Theorem 1.4 ([22] Theorem 1'). There is an effective constant $c_{3}$ such that

$$
\beta<\max \left\{1-\left(4 n_{K} !\left(\log d_{K}\right)\right)^{-1}, 1-\left(c_{3} d_{K}^{\frac{1}{n_{K}}}\right)^{-1}\right\} .
$$

Lemma 1.5. With $E_{4}=E_{4}(x, k)=\left(\log Q_{1}\right)\left(\log \log Q_{1}\right)\left(E_{1}(x, k)+E_{3}(x)\right)$, (with $E_{1}$ and $E_{3}$ as in Lemma 1.1 and Theorem 1.3 respectively) the following hold. 
1. $\tilde{r}_{k}\left(x, \delta_{C}\right) \ll E_{1}(x, k)+E_{3}(x)$.

2. $\sum_{q \leq Q_{1}}^{\prime} \max _{(a, q)=1} \max _{y \leq x}\left|\tilde{r}_{k}(y, \xi)\right| \ll \sum_{q \leq Q_{1}}^{\prime} \frac{1}{\phi(q)} \sum_{\chi \neq \chi_{0}} \max _{y \leq x}\left|\tilde{\psi}_{k}\left(y, \delta_{C} \otimes \chi\right)\right|+E_{4}$, where the final summation is over characters $\chi$ modulo $q$.

Proof. First we prove part 1. From Theorem 1.3, $r\left(x, \delta_{C}, K / M\right) \ll E_{3}$. This implies that

$$
\int_{1}^{x} r\left(t, \delta_{C}\right) \frac{d t}{t}=\int_{1}^{x}\left(\psi\left(t, \delta_{C}\right)-\frac{|C|}{|G|} t\right) \frac{d t}{t} \ll \int_{1}^{x}\left(\frac{|C|}{|G|} t^{\beta}+t \exp \left(-c n_{K}^{-\frac{1}{2}}(\log t)^{\frac{1}{2}}\right)\right) \frac{d t}{t} .
$$

Since the left hand side is $r_{1}\left(x, \delta_{C}\right)$, upon evaluating the integral, and using L'Hospital's rule on the quotient of $\int \exp \left(-c n_{K}^{-\frac{1}{2}}(\log t)^{\frac{1}{2}}\right) d t$ by $x \exp \left(-c n_{K}^{-\frac{1}{2}}(\log x)^{\frac{1}{2}}\right)$ for the last estimate,

$$
r_{1}\left(x, \delta_{C}\right) \ll \frac{|C|}{|G|} x^{\beta}+x \exp \left(-c n_{K}^{-\frac{1}{2}}(\log x)^{\frac{1}{2}}\right)=E_{3}(x) .
$$

Repeating the inverse Mellin transform implies that for any $k, r_{k}\left(x, \delta_{C}\right) \ll E_{3}(x)$. By Lemma 1.1 part 1 , $\tilde{r}_{k}\left(x, \delta_{C}\right) \ll E_{1}+E_{3}$.

Now we prove part 2. Combining the estimate in part 1 and (1.0.4),

$$
\tilde{r}_{k}(x, \xi) \ll \frac{1}{\phi(q)} \sum_{\chi \neq \chi_{0}} \bar{\chi}(a) \tilde{\psi}_{k}\left(x, \delta_{C} \otimes \chi\right)+\frac{1}{\phi(q)}\left(E_{1}+E_{3}\right) .
$$

Let $S=\sum_{q \leq Q_{1}}^{\prime} \max _{(a, q)=1} \max _{y \leq x}\left|\tilde{r}_{k}(y, \xi)\right|$. Therefore

$$
S \ll \sum_{q \leq Q_{1}}^{\prime} \frac{1}{\phi(q)} \sum_{\chi \neq \chi_{0}} \max _{y \leq x}\left|\tilde{\psi}_{k}\left(y, \delta_{C} \otimes \chi\right)\right|+\sum_{q \leq Q_{1}}^{\prime} \frac{1}{\phi(q)}\left(E_{1}+E_{3}\right) .
$$

Since $E_{1}$ and $E_{3}$ are independent of $q$, with the estimate $\sum_{q \leq Q_{1}} \phi(q)^{-1} \ll\left(\log Q_{1}\right)\left(\log \log Q_{1}\right)$ (see [11] $\S 18.4$ ), we conclude that

$$
S \ll \sum_{q \leq Q_{1}}^{\prime} \frac{1}{\phi(q)} \sum_{\chi \neq \chi_{0}} \max _{y \leq x}\left|\tilde{\psi}_{k}\left(y, \delta_{C} \otimes \chi\right)\right|+\left(\log Q_{1}\right)\left(\log \log Q_{1}\right)\left(E_{1}+E_{3}\right) .
$$

Now it suffices to estimate $\sum_{q \leq Q_{1}}^{\prime} \frac{1}{\phi(q)} \sum_{\chi \neq \chi_{0}} \max _{y \leq x}\left|\tilde{\psi}_{k}\left(y, \delta_{C} \otimes \chi\right)\right|$. The next step in our refinement is to replace the character sum by the sum over primitive, non-trivial characters. We indicate this restriction on a summation by the decoration $*$.

Lemma 1.6. With $E_{5}=E_{5}\left(x, k, Q_{1}\right)=\frac{1}{k !} Q_{1} \log Q_{1}(\log x)^{k+1}$,

$$
\sum_{q \leq Q_{1}}^{\prime} \frac{1}{\phi(q)} \sum_{\chi \neq \chi_{0}} \max _{y \leq x}\left|\tilde{\psi}_{k}\left(y, \delta_{C} \otimes \chi\right)\right| \ll\left(\log Q_{1}\right) \sum_{q \leq Q_{1}}^{\prime} \frac{1}{\phi(q)} \sum_{\chi}{ }^{*} \max _{y \leq x}\left|\tilde{\psi}_{k}\left(y, \delta_{C} \otimes \chi\right)\right|+E_{5} .
$$

Proof. By definition (0.6.1), since $\chi\left(\sigma_{p}^{m}\right)=\chi\left(N \mathfrak{p}^{m}\right)$ when considered as a Dirichlet character,

$$
\tilde{\psi}_{k}\left(x, \delta_{C} \otimes \chi\right)=\frac{1}{k !} \sum_{N \mathfrak{p}^{m} \leq x}(\log N \mathfrak{p})\left(\log \frac{x}{N \mathfrak{p}^{m}}\right)^{k} \delta_{C}\left(\sigma_{\mathfrak{p}}^{m}\right) \chi\left(N \mathfrak{p}^{m}\right) .
$$

If $\chi$ is a character modulo $q$ induced by the character $\chi^{*}$ modulo $q^{*}$ with $q^{*} \mid q$ and $q \neq q^{*}$ then

$$
\tilde{\psi}_{k}\left(x, \delta_{C} \otimes \chi\right)-\tilde{\psi}_{k}\left(x, \delta_{C} \otimes \chi^{*}\right)=\frac{1}{k !} \sum_{N \mathfrak{p}^{m} \leq x}(\log N \mathfrak{p})\left(\log \frac{x}{N \mathfrak{p}^{m}}\right)^{k} \delta_{C}\left(\sigma_{\mathfrak{p}}^{m}\right)\left(\chi\left(N \mathfrak{p}^{m}\right)-\chi^{*}\left(N \mathfrak{p}^{m}\right)\right) .
$$


The characters $\chi\left(N \mathfrak{p}^{m}\right)=\chi^{*}\left(N \mathfrak{p}^{m}\right)$ unless $N \mathfrak{p}^{m}$ divides $q / q^{*}$. Here $\chi(q)=0$ (by the definition of nonprimitive character). Therefore

$$
\begin{aligned}
\tilde{\psi}_{k}\left(x, \delta_{C} \otimes \chi\right)-\tilde{\psi}_{k}\left(x, \delta_{C} \otimes \chi^{*}\right) & =\frac{1}{k !} \sum_{N \mathfrak{p}^{m} \mid \frac{q}{q^{*}}}(\log N \mathfrak{p})\left(\log \frac{x}{N \mathfrak{p}^{m}}\right)^{k} \delta_{C}\left(\sigma_{\mathfrak{p}}^{m}\right)\left(\chi\left(N \mathfrak{p}^{m}\right)-\chi^{*}\left(N \mathfrak{p}^{m}\right)\right) \\
& \ll \frac{1}{k !}(\log x)^{k}\left(\log \frac{q}{q^{*}}\right) \ll \frac{1}{k !}(\log x)^{k+1} .
\end{aligned}
$$

For a fixed modulus $q$,

$$
\frac{1}{\phi(q)} \sum_{\chi \neq \chi_{0}} \max _{y \leq x}\left|\tilde{\psi}_{k}\left(y, \delta_{C} \otimes \chi\right)\right|=\sum_{\chi \neq \chi_{0}} \frac{1}{\phi(q)} \max _{y \leq x}\left|\tilde{\psi}_{k}\left(y, \delta_{C} \otimes \chi^{*}\right)\right|+O\left(\frac{1}{k !}(\log x)^{k+1} \sum_{\chi}{ }^{\circ} \frac{1}{\phi(q)}\right)
$$

where $\chi^{*}$ is the primitive character of modulus $q^{*}$ which induces $\chi$ and the decoration $\circ$ on the second summation indicates that the summation is over only those characters not primitive to the modulus $q$. Let $S=\sum_{q \leq Q_{1}}^{\prime} \sum_{\chi \neq \chi_{0}} \frac{1}{\phi(q)} \max _{y \leq x}\left|\tilde{\psi}_{k}\left(y, \delta_{C} \otimes \chi\right)\right|$. Then, it follows that

$$
S=\sum_{q \leq Q_{1}}^{\prime} \sum_{\chi \neq \chi_{0}} \frac{1}{\phi(q)} \max _{y \leq x}\left|\tilde{\psi}_{k}\left(y, \delta_{C} \otimes \chi^{*}\right)\right|+O\left(\frac{1}{k !}(\log x)^{k+1} \sum_{q \leq Q_{1}}^{\prime} \sum_{\chi(q)}^{\circ} \frac{1}{\phi(q)}\right) .
$$

Rewriting this as a sum over the conductors, (instead over the moduli of the non-primitive characters),

$$
S=\sum_{q^{*} \leq Q_{1}}^{\prime} \sum_{\chi^{*}\left(q^{*}\right)}^{*} \max _{y \leq x}\left|\tilde{\psi}_{k}\left(y, \delta_{C} \otimes \chi^{*}\right)\right| \sum_{\substack{q^{*} \mid q \\ q \leq Q_{1}}} \frac{1}{\phi(q)}+O\left(\frac{1}{k !}(\log x)^{k+1} \sum_{q^{*} \leq Q_{1}}^{\prime} \sum_{\chi^{*}\left(q^{*}\right)} * \sum_{\substack{q^{*} \mid q \\ q \leq Q_{1}}} \frac{1}{\phi(q)}\right)
$$

and since $\sum_{\substack{q^{*} \mid q \\ q \leq Q_{1}}} \frac{1}{\phi(q)} \ll \frac{\log Q_{1}}{\phi\left(q^{*}\right)}$ (see [7] Chapter 28 page 163),

$$
S \ll \log Q_{1} \sum_{q^{*} \leq Q_{1}}^{\prime} \sum_{\chi^{*}\left(q^{*}\right)}^{*} \frac{1}{\phi\left(q^{*}\right)} \max _{y \leq x}\left|\tilde{\psi}_{k}\left(y, \delta_{C} \otimes \chi^{*}\right)\right|+\frac{1}{k !}(\log x)^{k+1} \log Q_{1} \sum_{q^{*} \leq Q_{1}} \sum_{\chi\left(q^{*}\right)}^{*} \frac{1}{\phi\left(q^{*}\right)} .
$$

Since there are $\phi\left(q^{*}\right)$ characters modulo $q^{*}$, the right hand term is of the order

$$
\frac{1}{k !}(\log x)^{k+1} \log Q_{1} \sum_{q^{*} \leq Q_{1}} 1 \ll \frac{1}{k !}(\log x)^{k+1} Q_{1} \log Q_{1} \ll E_{5}
$$

We sum over dyadic intervals and use a standard estimate to replace the $\phi(q)$ in the summation in Lemma 1.6 with a $Q$, before at last combining the estimates of this section.

\section{Lemma 1.7.}

$$
\sum_{q \leq Q_{1}}^{\prime} \frac{1}{\phi(q)} \sum_{\chi}^{*} \max _{y \leq x}\left|\tilde{\psi}_{k}\left(y, \delta_{C} \otimes \chi\right)\right| \ll\left(\log Q_{1}\right)\left(\log \log Q_{1}\right) \max _{1 \leq Q \leq Q_{1}} \frac{1}{Q} \sum_{q \leq Q} \sum_{\chi}{ }^{*} \max _{y \leq x}\left|\tilde{\psi}_{k}\left(y, \delta_{C} \otimes \chi\right)\right| .
$$

Proof. Let $S$ be the double summation on the left side of the asymptotic in the statement of the lemma. Break $1 \leq q \leq Q_{1}$ into intervals of the form $\left(\frac{Q}{2}, Q\right]$, so

$$
S=\sum_{n} \sum_{q \in\left(\frac{Q}{2}, Q\right]} \frac{1}{\phi(q)} \max _{y \leq x} \sum_{\chi}^{*}\left|\tilde{\psi}_{k}\left(y, \delta_{C} \otimes \chi\right)\right|
$$


where $n$ ranges from 0 to $\left[\log _{2} Q_{1}\right]$ and $Q=2^{n+1}$. Since there are $\log Q_{1}$ of these intervals

$$
S \ll \log Q_{1} \max _{1 \leq Q \leq Q_{1}} \sum_{q \in\left(\frac{Q}{2}, Q\right]} \frac{1}{\phi(q)} \max _{y \leq x} \sum_{\chi} *\left|\tilde{\psi}_{k}\left(y, \delta_{C} \otimes \chi\right)\right| .
$$

The bound $\frac{1}{\phi(q)} \ll \frac{\log \log q}{q}$ (see [11] 18.4$)$ gives the new estimate

$$
S \ll \log Q_{1} \max _{1 \leq Q \leq Q_{1}} \sum_{q \in\left(\frac{Q}{2}, Q\right]} \frac{\log \log q}{q} \max _{y \leq x} \sum_{\chi}^{*}\left|\tilde{\psi}_{k}\left(y, \delta_{C} \otimes \chi\right)\right| .
$$

Since $\frac{Q}{2}<q \leq Q$ and $Q \leq Q_{1}$,

$$
S \ll\left(\log Q_{1}\right)\left(\log \log Q_{1}\right) \max _{1 \leq Q \leq Q_{1}} \frac{1}{Q} \sum_{q \in\left(\frac{Q}{2}, Q\right]} \max _{y \leq x} \sum_{\chi}^{*}\left|\tilde{\psi}_{k}\left(y, \delta_{C} \otimes \chi\right)\right|
$$

which is clearly less than the summation over the expanded range $q \leq Q$.

We combine Lemmas 1.2, 1.5 part 2, 1.6, and 1.7 to transform the average into a more usable form.

Proposition 1.8. Let $K / M$ be a Galois extension of number fields, with group $G$ and let $C$ be a conjugacy class in $G$. Let $H$ be an abelian subgroup of $G$ such that $H \cap C \neq \emptyset$ and let $E$ be the fixed field of $H$. For any $\epsilon>0$ and $Q_{1} \leq x^{\frac{1}{2}-\epsilon}$ in order to prove that for any $D>0$

$$
\sum_{q \leq Q_{1}}^{\prime} \max _{(a, q)=1} \max _{y \leq x}\left|r_{0}(y, C, q, a)\right| \ll \frac{x}{(\log x)^{D}}
$$

it suffices to prove either of the following

1. For all $1 \leq Q \leq Q_{1}$ and any $F>0$, there is a $k \geq 0$ such that

$$
\frac{1}{Q} \sum_{q \leq Q}^{\prime} \sum_{\chi}^{*} \max _{y \leq x}\left|\tilde{\psi}_{k}\left(y, \delta_{C} \otimes \chi, K\left(\zeta_{q}\right) / M\right)\right| \ll \frac{x}{(\log x)^{F}}
$$

where the second summation is over all primitive non-trivial Dirichlet characters of modulus $q$.

2. For all $1 \leq Q \leq Q_{1}$ and any $F>0$, there is a $k \geq 0$ such that

$$
\frac{1}{Q} \sum_{q \leq Q}^{\prime} \sum_{\omega}^{*} \max _{y \leq x}\left|\tilde{\psi}_{k}\left(y, \omega, K\left(\zeta_{q}\right) / E\right)\right| \ll \frac{x}{(\log x)^{F}}
$$

where the second summation is over irreducible non-trivial characters $\omega$ of $\operatorname{Gal}\left(K\left(\zeta_{q}\right) / E\right)$ such that $A(\omega) \ll A q^{n_{E}}$ where $A$ is the conductor of $L(s, K / M)$. Moreover, one may assume that there are $O(\phi(q))$ characters $\omega$ in the second summation.

Proof. First, we prove part 1. Let $E_{2}=E_{2}(x, k, 0, A),\left(\right.$ as in Lemma 1.2) $f=\exp \left(k(\log x)^{-A}\right), A^{\prime}=$ $\frac{1}{2} k(k+1) A$, and $S=\sum_{1 \leq Q_{1}}^{\prime} \max _{(a, q)=1} \max _{y \leq x}\left|r_{0}(y, C, q, a)\right|$. We will show that for every $B>0$

$$
S \ll(\log x)^{A^{\prime}+3} \max _{1 \leq Q \leq Q_{1}} \frac{1}{Q} \sum_{q \leq Q}^{\prime} \sum_{\chi}^{*} \max _{y \leq x f}\left|\tilde{\psi}_{k}\left(y, \delta_{C} \otimes \chi\right)\right|+\frac{x}{(\log x)^{B}} .
$$

This proves the proposition as we can replace $x f$ with $x$ in the maximum since $1 \leq f \leq\left(e^{k !}\right)$ when $x \geq 2$. It then suffices to choose $F>D+A^{\prime}+3$. 
We now estimate $S$.

$$
\begin{array}{lr}
S \ll(\log x)^{A^{\prime}} \sum_{q \leq Q_{1}}^{\prime} \max _{(a, q)=1} \max _{y \leq x f}\left|\tilde{r}_{k}(y, \xi)\right|+E_{2} & \text { Lemma } 1.2 \text { part } 2 \\
\ll(\log x)^{A^{\prime}} \sum_{q \leq Q_{1}}^{\prime} \frac{1}{\phi(q)} \sum_{\chi}^{*} \max _{y \leq x f}\left|\tilde{\psi}_{k}\left(y, \delta_{C} \otimes \chi\right)\right|+E_{4}(x f)+E_{2} & \text { Lemma } 1.5 \text { part } 2 \\
\ll(\log x)^{A^{\prime}} \log Q_{1} \sum_{q \leq Q_{1}}^{\prime} \frac{1}{\phi(q)} \sum_{\chi}^{*} \max _{y \leq x f}\left|\tilde{\psi}_{k}\left(y, \delta_{C} \otimes \chi\right)\right|+E_{5}(x f)+E_{4}(x f)+E_{2} & \text { Lemma } 1.6 \\
\ll(\log x)^{A^{\prime}}\left(\log Q_{1}\right)^{2}\left(\log \log Q_{1}\right) \max _{1 \leq Q \leq Q_{1}} \frac{1}{Q} \sum_{q \leq Q}^{\prime} \sum_{\chi}^{*} \max _{y \leq x f}\left|\tilde{\psi}_{k}\left(y, \delta_{C} \otimes \chi\right)\right| & \text { Lemma } 1.7
\end{array}
$$

Let $A^{\prime \prime}=\left(\frac{1}{2} k A+1\right)(k+1) A$. Since $Q_{1} \leq x^{\frac{1}{2}-\epsilon}, f$ is bounded, and from Theorem 1.4, $\beta<\{1-$ $\left.\left(4 n_{K} !\left(\log d_{K}\right)\right)^{-1}, 1-\left(c_{3} d_{K}^{\frac{1}{n_{K}}}\right)^{-1}\right\}$, by direct analysis the error

$$
\begin{aligned}
E_{5}(x f)+E_{4}(x f)+E_{2} & \ll x^{1-\epsilon}(\log x)^{A^{\prime \prime}}+x(\log x)^{2-A}+x^{\beta}(\log x)^{2}+x(\log x)^{2} \exp \left(-c n_{K}^{-\frac{1}{2}}(\log x f)^{\frac{1}{2}}\right) \\
& \ll x(\log x)^{-D}
\end{aligned}
$$

choosing $A-2 \leq D$. (Note that $\exp \left(-c n_{K}^{-\frac{1}{2}}(\log x f)^{\frac{1}{2}}\right) \ll(\log x)^{B}$ for any $B>0$.) This proves part 1 .

Now we prove part 2. By the equation before (0.7.1)

$$
\tilde{\psi}_{k}\left(x, \delta_{C} \otimes \chi, K\left(\zeta_{q}\right) / M\right)=\frac{|C|}{|G|} \sum_{\eta} \bar{\eta}(g) \tilde{\psi}_{k}\left(x, \eta \otimes \chi, K\left(\zeta_{q}\right) / E\right)
$$

where the summation is over irreducible characters $\eta$ of $G$. Since $\eta$ is irreducible, for $\chi$ a primitive character of modulus $q, \eta \otimes \chi$ is also irreducible. By $\S 0.4$, if $\omega=\eta \otimes \chi$ then $A(\omega)=A(\eta \otimes \chi)=A(\eta) A(\chi) \ll A(\eta) A q^{n_{E}}$, which is $O\left(A q^{n_{E}}\right)$ with the implied constant depending only on $K$. Therefore,

$$
\tilde{\psi}_{k}\left(x, \delta_{C} \otimes \chi, K\left(\zeta_{q}\right) / M\right) \ll \frac{|C|}{|G|} \sum_{\omega}\left|\tilde{\psi}_{k}\left(x, \omega, K\left(\zeta_{q}\right) / E\right)\right|
$$

where the sum is over irreducible $\omega$ of $\operatorname{Gal}\left(K\left(\zeta_{q}\right) / E\right)$ such that $A(\omega) \ll A q^{n_{E}}$. Since this is a finite sum over the $\omega$ by part 1 it suffices to bound the maximum.

Definition 1.1. For $K / M$ Galois and $C$ a conjugacy class of $G=\operatorname{Gal}(K / M)$, let $H$ be an abelian subgroup of $G$ so that $H \cap C \neq \emptyset$ and let $E$ be the fixed field of $H$. Letting $H$ be the largest such subgroup, define $d=n_{E}$.

We will prove the above estimate for small values of $Q$, those at most $(\log x)^{\gamma}$, in Proposition 2.1 and for large values of $Q$, those between $(\log x)^{\gamma}$ and $\min \left\{x^{\frac{1}{2}-\epsilon}, x^{\frac{1}{d-2}-\epsilon}\right\}$, in Proposition 3.1.

\section{The Initial Range}

By Proposition 1.8 part 2 we can reduce to the case of $K / E$ abelian, and consider the functions $\tilde{\psi}\left(x, \omega, K\left(\zeta_{q}\right) / E\right)$ where $\omega$ is a non-trivial irreducible Hecke character of $\operatorname{Gal}\left(K\left(\zeta_{q}\right) / E\right)$ and $\psi=\psi_{0}$. We will assume that $K / E$ is abelian for the remainder of the section and that $A(\omega) \ll A q^{n_{E}}$ where $A$ is the conductor of $L(s, K / M)$, which we can do without loss of generality by Proposition 1.8 part 2. We let $L=K\left(\zeta_{q}\right)$. We will suppress the notation of $K\left(\zeta_{q}\right) / E$ in writing $\psi$ functions and associated $L$-functions unless needed for clarity.

This section will be devoted to the proof of the following proposition. 
Proposition 2.1 (Initial Range). Let $K / E$ be an abelian Galois extension, and let $D$ and $\gamma$ be any positive constants. For all $Q \leq(\log x)^{\gamma}$ we have

$$
\frac{1}{Q} \sum_{q \leq Q} \sum_{\omega}^{*} \max _{y \leq x}|\tilde{\psi}(y, \omega)| \ll \frac{x}{(\log x)^{D}}
$$

where the inner summation is over all irreducible, non-trivial characters $\omega$ of $K\left(\zeta_{q}\right) / E$ such that $A(\omega) \ll A q^{d}$.

Proposition 2.1 follows from the following proposition.

Proposition 2.2. For $K / E$ abelian, there is a positive constant $c=c(K, E)$ so that for any non-trivial irreducible character $\omega$ of $\operatorname{Gal}\left(K\left(\zeta_{q}\right) / E\right)$

$$
|\tilde{\psi}(x, \omega)| \ll(\log A(\omega)) x \exp \left(-c(\log x)^{\frac{1}{2}}\right) .
$$

Before proving Proposition 2.2, we show that it implies Proposition 2.1.

Proof of Proposition 2.1. Let $D>0$ be fixed. By Proposition 2.2, there is a $c>0$ so that

$$
|\tilde{\psi}(x, \omega)| \ll\left(\log A q^{n_{E}}\right) x \exp \left(-c(\log x)^{\frac{1}{2}}\right) .
$$

Assume that $Q \leq(\log x)^{\gamma}$, then for some positive $N, A(\omega) \ll A q^{n_{E}} \ll(\log x)^{N}$. We have,

$$
\begin{aligned}
\frac{1}{Q} \sum_{q \leq Q} \sum_{\chi}{ }^{*} \max _{y \leq x}|\tilde{\psi}(y, \omega)| & \ll \frac{1}{Q} \sum_{q \leq Q} \phi(q)(\log A(\omega)) x \exp \left(-c(\log x)^{\frac{1}{2}}\right) \\
& \ll Q(\log x)^{N} x \exp \left(-c(\log x)^{\frac{1}{2}}\right) \\
& \ll(\log x)^{\gamma+N} x \exp \left(-c(\log x)^{\frac{1}{2}}\right) \\
& \ll x(\log x)^{-D} .
\end{aligned}
$$

Therefore it suffices to prove Proposition 2.2.

The remainder of the section will be devoted to the proof of Proposition 2.2. In $\S 2.1$ we prove preliminary results about zero free regions and Siegel zeros. In the following section, $\S 2.2$, we provide the framework to prove Proposition 2.2 by connecting $\tilde{\psi}(x, \omega)$ with the zeros of $L(s, \omega)$ using the method of contour integration. Finally, in $\S 2.3$, we prove Proposition 2.2 .

\subsection{Zero Free Regions}

We wish to bound $\tilde{\psi}\left(x, \omega, K\left(\zeta_{q}\right) / E\right)$, With (0.6.2) we will do so by showing the existence of a region with at most one zero for $L(s, \omega)$ and provide bounds for this possible zero. This closely follows the bounds in [17] and [22]. In what follows, we use the notation $s=\sigma+i t$.

Definition 2.1. For $K / E$ abelian and parameter $t$, let $\mathcal{L}(t)=\frac{1}{2} \log A(\omega)+n_{E} \log (|t|+2)$.

Since $A(\omega) \ll A q^{d}, \mathcal{L}(t) \ll \frac{1}{2} \log \left(A q^{d}\right)+n_{E} \log (|t|+2)$. We now compile some facts, mainly from [13] (pages 432-434) and $\S 0.7$. We will assume that $\omega$ is an irreducible non-trivial Artin character. First, we prove the following lemma.

Lemma 2.3. Let $s=\sigma+i$. For a constant $C_{1}$ with $1<\sigma<C_{1}$ and non-trivial irreducible $\omega$ there is a positive absolute constant $C_{2}$ such that

$$
-\operatorname{Re} \frac{L^{\prime}}{L}(s, \omega)<C_{2} \mathcal{L}(t)-\sum_{\rho} \operatorname{Re}\left(\frac{1}{s-\rho}\right)
$$


Proof. By (0.7.3)

$$
2 \operatorname{Re} \frac{L^{\prime}}{L}(s, \omega)=\sum_{\rho}\left(\frac{1}{s-\rho}+\frac{1}{s-\bar{\rho}}\right)+\operatorname{Re}\left(-\frac{1}{2} \log A(\omega)-\frac{\gamma^{\prime}}{\gamma}(s, \omega)\right) .
$$

Stirling's formula, for $\sigma>1$ (see [13] Lemma 5.3) implies that $\operatorname{Re} \frac{\gamma^{\prime}}{\gamma}(s, \omega) \ll n_{E} \log (|t|+2)$ which proves the result.

The following proposition gives a region with at most one zero, which must be real and simple. Proposition 2.8 gives more precise bounds for such zero. Propositions 2.4 and 2.8 will be used in the proof of Proposition 2.2 .

Proposition 2.4. There is an absolute positive constant $C$ such that $L(s, \omega)$ has at most one zero $\sigma+i t$ in the region

$$
1-\frac{C}{\mathcal{L}(t)} \leq \sigma \leq 1
$$

If such a zero exists, then it is real and simple and $\omega$ is a character of order 1 or 2. (That is, $\omega$ is a quadratic Hecke character, since $\omega$ is assumed not to be the trivial character.)

We will call this zero $\beta$ if it exists, and refer to $\beta$ as a Siegel zero of $L(s, \omega)$. By the functional equation (0.4.3) this implies that there is at most one zero of $L(s, \omega)$ in the region $0 \leq \sigma \leq C / \mathcal{L}(t)$ as well. This zero, $1-\beta$, must be simple as well. If the constant $C$ is large enough that $1-C / \mathcal{L}(0) \leq \frac{1}{2}$, then Proposition 2.4 implies that there can be no zeros in the range $1-C / \mathcal{L}(t) \leq \sigma \leq 1$. If $D<C$ the region $1-D / \mathcal{L}(t)$ is contained in the region $1-C / \mathcal{L}(t)$. Therefore, we can assume that $C<\frac{1}{2} \mathcal{L}(0)=\frac{1}{2}\left(\frac{1}{2} \log A(\omega)+n_{E} \log 2\right)$.

Proof. We will use the notation $\rho=\beta+i \gamma$ for zeros and $s=\sigma+i$ for a complex parameter. First, we will collect some useful facts. Recall that (if we extend $\omega$ multiplicatively) from (0.3.2)

$$
-\frac{L^{\prime}}{L}(s, \omega)=\sum_{\mathfrak{p}} \sum_{m=1}^{\infty} \frac{(\log N \mathfrak{p}) \omega\left(\sigma_{\mathfrak{p}}^{m}\right)}{N \mathfrak{p}^{m s}}=\sum_{\mathfrak{a}} \frac{\Lambda(N \mathfrak{a}) \omega(\mathfrak{a})}{N \mathfrak{a}^{s}}=\sum_{\mathfrak{a}} \Lambda(N \mathfrak{a}) \omega(\mathfrak{a})(N \mathfrak{a})^{-\sigma} e^{-i t(\log N \mathfrak{a})}
$$

where the first sum is over prime ideals, $\mathfrak{p}$ of $E, N=N_{E / \mathbb{Q}}$ and the last sum is over ideals $\mathfrak{a}$ of $E$. We can rewrite the trigonometric inequality (which holds for $\theta \in \mathbb{R}$ )

$$
3+4 \cos \theta+\cos 2 \theta=2(1+\cos \theta)^{2} \geq 0
$$

with $\cos \theta=\operatorname{Re} \omega(\mathfrak{a}) e^{-i t \log N \mathfrak{a}}$. With $\omega_{0}$ denoting the principal character of conductor $\mathfrak{f}(\omega)$ we have (See [7] $\S 14$ page 88, [2] page 195.)

$$
\left[-3 \operatorname{Re} \frac{L^{\prime}}{L}\left(\sigma, \omega_{0}\right)-4 \operatorname{Re} \frac{L^{\prime}}{L}(\sigma+i t, \omega)-\operatorname{Re} \frac{L^{\prime}}{L}\left(\sigma+2 i t, \omega^{2}\right)\right] \geq 0 .
$$

By Lemma 3 of [22], for a number field $F$ with $r_{1}$ real and $r_{2}$ complex places and $s=\sigma+i t$ with $\sigma>1$

$$
-\operatorname{Re} \frac{\zeta_{F}^{\prime}}{\zeta_{F}}(s)<\operatorname{Re} \frac{1}{s}+\operatorname{Re} \frac{1}{s-1}+\frac{1}{2} \log \left(\frac{d_{F}}{2^{2 r_{2}} \pi^{n_{F}}}\right)+\frac{r_{1}}{2} \operatorname{Re} \frac{\Gamma^{\prime}}{\Gamma}\left(\frac{s}{2}\right)+r_{2} \operatorname{Re} \frac{\Gamma^{\prime}}{\Gamma}(s) .
$$

Also, from Lemma 1 of [22] (with $f(s)=s(s-1) \zeta_{F}(s)$ )

$$
\sum_{\rho} \frac{1}{s-\rho}=\frac{1}{s-1}+\frac{1}{2} \log d_{F}+\left(\frac{1}{s}-\frac{n_{F}}{2} \log \pi\right)+\frac{r_{1}}{2} \frac{\Gamma^{\prime}}{\Gamma}\left(\frac{s}{2}\right)+r_{2}\left(\frac{\Gamma^{\prime}}{\Gamma}(s)-\log 2\right)+\frac{\zeta_{F}^{\prime}}{\zeta_{F}}(s) .
$$

If $s=\sigma$ with $1<\sigma<2$ then all terms after $\frac{1}{2} \log d_{F}$ are negative, and the left hand side is positive. Therefore,

$$
-\frac{\zeta_{F}^{\prime}}{\zeta_{F}}(\sigma) \leq \frac{1}{\sigma-1}+\frac{1}{2} \log d_{F}
$$


The proof will be based on estimates for the terms in (2.1.1), heavily relying on (2.1.2) and (2.1.3). We will now estimate the first two terms of (2.1.1). Writing out the summation for $\sigma>1$ by direct comparison of terms,

$$
-\frac{L^{\prime}}{L}\left(\sigma, \omega_{0}\right) \leq-\frac{\zeta_{E}^{\prime}}{\zeta_{E}}(\sigma)
$$

By this and (2.1.3),

$$
-\frac{L^{\prime}}{L}\left(\sigma, \omega_{0}\right)<\frac{1}{\sigma-1}+C_{1} \mathcal{L}(0)
$$

for some positive constant $C_{1}$. For any $s=\sigma+i$ and zero $\rho=\beta+i \gamma$ in the range $0<\beta<1<\sigma$ we have

$$
\operatorname{Re}\left(\frac{1}{s-\rho}\right)=\frac{\sigma-\beta}{|s-\rho|^{2}} \geq 0 .
$$

Due to this positivity, Lemma 2.3 implies that for such a $\rho$, there is a positive absolute constant $C_{2}$ so that

$$
-\operatorname{Re} \frac{L^{\prime}}{L}(s, \omega)<C_{2} \mathcal{L}(t)-\operatorname{Re}\left(\frac{1}{s-\rho}\right) .
$$

Choose $t=\gamma$, so that $\operatorname{Re} 1 /(s-\rho)=1 /(\sigma-\beta)$. Combining this and (2.1.4) into inequality (2.1.1) there is a positive $C_{3}$ so that

$$
\frac{4}{\sigma-\beta}<C_{3} \mathcal{L}(t)+\frac{3}{\sigma-1}-\operatorname{Re} \frac{L^{\prime}}{L}\left(\sigma+2 i t, \omega^{2}\right) .
$$

It remains to estimate this remaining $-L^{\prime} / L$ term.

Many of the auxiliary results we will use require $\omega^{2}$ to be irreducible. If $\omega^{2}$ is induced by $\omega^{*}$, the difference between the corresponding $L^{\prime} / L$ factors is small. This difference is

$$
\begin{aligned}
\operatorname{Re}\left|\frac{L^{\prime}}{L}\left(s, \omega^{2}\right)-\frac{L^{\prime}}{L}\left(s, \omega^{*}\right)\right| & =\operatorname{Re}\left|\sum_{\mathfrak{p} \mid \mathfrak{f}(\omega)} \sum_{m=1}^{\infty} \frac{(\log N \mathfrak{p}) \omega\left(\sigma_{\mathfrak{p}}^{m}\right)}{N \mathfrak{p}^{m s}}\right| \\
& \leq \log N \mathfrak{f}(\omega) \sum_{\mathfrak{p} \mid \mathfrak{f}(\omega)} \sum_{m=1}^{\infty} N \mathfrak{p}^{-m \sigma} \\
& \leq \log A(\omega) \sum_{\mathfrak{p} \mid \mathfrak{f}(\omega)}\left(1+N \mathfrak{p}^{-\sigma}\right) \\
& \leq \log A(\omega) \sum_{\mathfrak{p} \mid d_{K}} 2 \leq 2 d_{K / E} \log A(\omega) \ll \mathcal{L}(t) .
\end{aligned}
$$

Therefore, replacing $\omega^{2}$ by $\omega^{*}$ in (2.1.6) only changes the positive constant in the equality.

The argument now breaks into two cases, depending on whether or not $\omega^{2}$ is a principal character. First, assume that $\omega^{2}$ is not a principal character. We may assume that $\omega^{2}$ is irreducible. By (2.1.5) $-\operatorname{Re} \frac{L^{\prime}}{L}\left(s, \omega^{2}\right)<C_{3} \mathcal{L}(t)$. Inequality (2.1.6) now becomes, for $1<\sigma<2$, and a positive $C_{4}$,

$$
\frac{4}{\sigma-\beta}<C_{4} \mathcal{L}(t)+\frac{3}{\sigma-1} .
$$

Writing $\sigma=1+D / \mathcal{L}(t)$, this is

$$
\beta<1+\frac{D}{\mathcal{L}(t)}-\frac{4 D}{\left(3+D C_{4}\right) \mathcal{L}(t)}=1-\frac{1}{\mathcal{L}(t)}\left(\frac{D\left(C_{4} D-1\right)}{C_{4} D+3}\right) .
$$

Therefore, if $D<C_{4}^{-1}$ we conclude that there is an absolute constant $C>0$ such that

$$
\beta<1-\frac{C}{\mathcal{L}(t)}
$$


This concludes the proof in the case where $\omega^{2}$ is not principal, as this is not in the prescribed range.

We now consider the case where $\omega^{2}$ is a principal character. As we can reduce to considering irreducible characters, we may assume that $\omega^{2}$ is the trivial character. Inequality (2.1.4) with the fact that $1<\sigma<2$ implies that there is a positive constant $D_{1}$ so that

$$
-\operatorname{Re} \frac{L^{\prime}}{L}\left(\sigma+2 i t, \omega^{2}\right)<\operatorname{Re}\left(\frac{1}{\sigma-1+2 i t}\right)+D_{1} \mathcal{L}(0) .
$$

Substituting this into (2.1.6), there is a positive $D_{2}$ so that

$$
\frac{4}{\sigma-\beta}<\operatorname{Re}\left(\frac{1}{\sigma-1+2 i t}\right)+D_{2} \mathcal{L}(t)+\frac{3}{\sigma-1} .
$$

With $\sigma=1+D / \mathcal{L}(t)$, if $t=\gamma>D / \mathcal{L}(t)$ we conclude that

$$
\frac{4}{\sigma-\beta}<\frac{16}{5} \frac{\mathcal{L}(t)}{D}+D_{2} \mathcal{L}(t)
$$

and therefore

$$
\beta<1-\frac{D}{\mathcal{L}(t)}\left(\frac{4-5 D_{2} D}{16+5 D_{2} D}\right) .
$$

For a sufficiently small $D$ depending on $D_{2}$, the value $D\left(4-5 D_{2} D\right) /\left(16+5 D_{2} D\right)$ is bounded below by a constant. Choosing $t=\gamma>D / \frac{1}{2} \log A(\omega)$ satisfies the bound $\gamma>D / \mathcal{L}(t)$.

We have shown that there are positive absolute constants $C$, and $D^{\prime}$ such that if $\omega$ is a non-trivial irreducible character, then any zero $\beta+i \gamma$ of $L(s, \omega)$ with $\gamma \geq D^{\prime} / \frac{1}{2} \log A(\omega)$ satisfies $\beta<1-C / \mathcal{L}(t)$.

It now remains to assume that $\omega^{2}$ is non-principal and

$$
|t|=|\gamma|<\frac{D}{\frac{1}{2} \log A(\omega)}
$$

for a small constant $D$. We wish to show that there is a positive constant $F$ such that there is at most one zero $\beta+i \gamma$ with $\beta>1-F / \mathcal{L}(t)$. It suffices to show that there is at most one zero with $\beta>1-F / \frac{1}{2} \log A(\omega)$ since $\frac{1}{2} \log A(\omega)<\mathcal{L}(t)$. Such a zero must be real, as otherwise there would be a pair of conjugate zeros.

By Lemma 2.3, for $\sigma>1$ there is a positive constant $F_{1}$ such that

$$
-\frac{L^{\prime}}{L}(\sigma, \omega)<F_{1} \log A(\omega)-\sum_{\rho} \frac{1}{\sigma-\rho}
$$

where the last summation is real as the zeros occur in complex conjugate pairs. We have,

$$
-\frac{L^{\prime}}{L}(\sigma, \omega)=\sum_{\mathfrak{a}} \omega\left(\sigma_{\mathfrak{a}}\right) \Lambda(N \mathfrak{a}) N \mathfrak{a}^{-\sigma} \geq-\sum_{\mathfrak{a}} \Lambda(N \mathfrak{a}) N \mathfrak{a}^{-\sigma} \geq \frac{\zeta_{E}^{\prime}}{\zeta_{E}}(\sigma) .
$$

Combining this with (2.1.3),

$$
-\frac{L^{\prime}}{L}(\sigma, \omega) \geq-\frac{1}{\sigma-1}-\frac{1}{2} \log d_{E} .
$$

Therefore, there is a positive constant $F_{2}$ so that

$$
-\frac{1}{\sigma-1} \leq F_{2} \log A(\omega)-\sum_{\rho} \frac{1}{\sigma-\rho} .
$$

Now we consider a few cases, depending on the type of possible zeros. Specifically, we will first assume that there is a complex zero (and hence its conjugate is also a zero) and then assume that there are at least two (possibly identical) real zeros. In each case, we will conclude that there is a positive constant $F$ such that the real parts of the zeros are less than $1-F / \mathcal{L}(t)$. Therefore, there is a positive constant $C$ such that there can be at most one zero with real part between $1-C / \mathcal{L}(t)$ and 1 . 
Assume that there are zeros $\beta \pm i \gamma$ with $\gamma \neq 0$. Inequality (2.1.7) implies that

$$
-\frac{1}{\sigma-1} \leq F_{2} \log A(\omega)-\frac{2(\sigma-\beta)}{(\sigma-\beta)^{2}+\gamma^{2}} .
$$

With $\sigma=1+2 D / \frac{1}{2} \log A(\omega)$ we have

$$
|\gamma|<\frac{D}{\frac{1}{2} \log A(\omega)}=\frac{1}{2}(\sigma-1)<\frac{1}{2}(\sigma-\beta) .
$$

Therefore there is a constant $F_{3}>0$ so that

$$
-\frac{1}{\sigma-1}<F_{3} \log A(\omega)-\frac{8}{5(\sigma-\beta)} .
$$

This is equivalent to

$$
\beta<\sigma-\frac{8}{5} \frac{\sigma-1}{1+F_{3} \frac{1}{2} \log A(\omega)(\sigma-1)}=1-\frac{2 D}{\frac{1}{2} \log A(\omega)}\left(\frac{3-10 D F_{3}}{1+2 D F_{3}}\right)
$$

we conclude that there is a positive constant $F$ so that $\beta<1-F / \mathcal{L}(t)$ if $3-10 D F_{3}$ is positive. Choosing $D<3 / 10 F_{3}$ suffices.

It remains to consider the case where there are at least two real zeros, $\beta_{1} \leq \beta_{2}$. Inequality (2.1.7) implies that

$$
-\frac{1}{\sigma-1} \leq F_{2} \log A(\omega)-\frac{2}{\sigma-\beta_{2}} .
$$

With $\sigma=1+2 D / \frac{1}{2} \log A(\omega)$ as before

$$
\beta<1-\frac{2 D}{\frac{1}{2} \log A(\omega)}\left(\frac{1-2 F_{2} D}{1+2 F_{2} D}\right)
$$

and if $D<1 / 2 F_{2}$ we conclude that there is a positive constant $F$ so that $\beta<1-F / \mathcal{L}(t)$.

The following lemma is a refinement of the Brauer-Siegel theorem, which gives a bound for $\beta$. We require a bound for $\beta$ that has minimal dependence on the field $L=K\left(\zeta_{q}\right)$. The following facilitates such a bound, with only a logarithm term depending on $q$. This is a refinement of Lemma 10 in [22].

Lemma 2.5. Let

$$
\mathbb{Q}=F_{0} \subset F_{1} \subset F_{2} \subset \cdots \subset F_{t}=F
$$

be a sequence of number fields such that for $2 \leq i \leq t, F_{i} / F_{i-1}$ is Galois. If there is a real $\beta$ in the range

$$
1-\frac{1}{4\left(2 n_{F_{1}}\right) ! \log d_{F}} \leq \beta<1
$$

such that $\zeta_{F}(\beta)=0$, then there is a quadratic field $S \subseteq F$ with $\zeta_{S}(\beta)=0$.

This proof will make use of Theorem 3, Lemma 3, and Lemma 8 of [22], which we summarize in the following theorem.

Theorem 2.6 (Stark).

a) If $K_{4} / K_{1}$ is a Galois extension of number fields and $\alpha$ is a simple zero of $\zeta_{K_{4}}$ then there is a field $K_{2}$ cyclic over $K_{1}$ and contained in $K_{4}$ such that for any field $K_{3}$ between $K_{1}$ and $K_{4}, \zeta_{K_{3}}(\alpha)=0$ if and only if $K_{2} \subset K_{3}$. If $\alpha$ is real then $K_{2}=K_{1}$ or is quadratic over $K_{1}$.

b) If $K$ is a number field then $\zeta_{K}$ has at most one zero $\sigma+i$ in the region $\sigma \geq 1-\left(4 \log d_{K}\right)^{-1}$, $|t| \leq\left(4 \log d_{K}\right)^{-1}$. If such a zero exists, it is real and simple. 
c) Let $K$ be a number field. If there is a real $\alpha$ in the range $1-\left(4 n_{K} ! \log d_{K}\right)^{-1} \leq \alpha<1$ such that $\zeta_{K}(\alpha)=0$, then there is a quadratic field $S$ contained in $K$ so that $\zeta_{S}(\alpha)=0$.

Proof of Lemma 2.5. We will first prove the following claim.

Claim 2.7. Let $A \subset B \subset C$ with $C / B$ and $B / A$ Galois. Assume that there is a real $\beta$ such that $1-$ $\left(4 \log d_{C}\right)^{-1} \leq \beta<1$ and $\zeta_{C}(\beta)=0$. Either $\zeta_{A}(\beta)=0$ or there is a quadratic extension $A_{1}$ of $A$ with $A_{1} \subset C$ such that $\zeta_{A_{1}}(\beta)=0$.

First, we show that Claim 2.7 implies Lemma 2.5. Since $1-\left(4\left(2 n_{1}\right) ! \log d_{F}\right)^{-1} \leq \beta$ and $4 \log d_{F} \leq$ $4\left(2 n_{1}\right) ! \log d_{F}, 1-\left(4 \log d_{F}\right)^{-1} \leq \beta$. By Claim $2.7 \zeta_{F_{t-2}}(\beta)=0$ or there is a quadratic extension, $Q_{t-1}$ of $F_{t-2}$ such that $\zeta_{Q_{t-1}}(\beta)=0$. Since both $4 \log d_{Q_{t-1}}$ and $4 \log d_{F_{t-2}}$ are less than $4 \log d_{F}$ we can proceed inductively and conclude that $\zeta_{F_{1}}(\beta)=0$ or there is a quadratic extension $Q_{2}$ of $F_{1}$ such that $\zeta_{Q_{2}}(\beta)=0$. Let $S^{\prime}$ be this field. Therefore, $n_{S^{\prime}} \leq 2 n_{1}$. Discriminant relations imply that $d_{S^{\prime}} \leq d_{F}$ so that $4 n_{S^{\prime}}$ ! log $d_{S^{\prime}} \leq$ $4\left(2 n_{1}\right) ! \log d_{S^{\prime}} \leq 4\left(2 n_{1}\right) ! \log d_{F}$. Therefore $1-\left(4 n_{S^{\prime}} ! \log d_{S^{\prime}}\right)^{-1} \leq 1-\left(4\left(2 n_{1}\right) ! \log \bar{d}_{F}\right)^{-1} \leq \beta$ and by Theorem $2.6 \mathrm{c})$ there is a quadratic field $S \subset F$ such that $\zeta_{s}(\beta)=0$.

Proof of Claim 2.7. By Theorem $2.6 \mathrm{~b}), \beta$ is simple. By Theorem 2.6 a) either $\zeta_{B}(\beta)=0$ or there is a quadratic extension $B_{1}$ of $B$ contained in $C$ such that $\zeta_{B_{1}}(\beta)=0$. If $\zeta_{B}(\beta)=0$ we can apply Theorem 2.6 b) and a) again and conclude that either $\zeta_{A}(\beta)=0$ or that there is a quadratic extension $A_{1}$ of $A$ contained in $B \subset C$ such that $\zeta_{A_{1}}(\beta)=0$. Otherwise, assume that $\zeta_{B_{1}}(\beta)=0$ and $\zeta_{B}(\beta) \neq 0$. If $B_{1}$ is Galois over $A$ we can reapply Theorem $2.6 \mathrm{~b}$ ) and a) to again conclude that either $\zeta_{A}(\beta)=0$ or there is a quadratic extension $A_{1}$ of $A$ contained in $B_{1} \subset C$ such that $\zeta_{A_{1}}(\beta)=0$. Therefore, it suffices to show that $B_{1}$ is Galois over $A$.

We will assume that $B_{1}$ is not Galois over $A$. By Theorem 2.6 a), for any field $E$ between $B_{1}$ and $C, \zeta_{E}(\beta)=0$. Let $B_{1}^{\prime}$ be the conjugate of $B_{1}$ over $A$, so $\left[B_{1}^{\prime}: B\right]=2$ as $B / A$ is Galois. Both $B_{1}$ and $B_{1}^{\prime}$ are Galois over $B$, so the composite $B_{2}=B_{1} B_{1}^{\prime}$ is Galois and degree 4 over $B$. Since $B_{1}^{\prime}$ is the $A$ conjugate of $B_{1}, \zeta_{B_{1}^{\prime}}(\beta)=\zeta_{B_{1}}(\beta)=0$. By the Aramata Brauer theorem $[1,5] \zeta_{B_{2}}(\beta)=0$. In fact $($ see [22] Theorem 3, or Lemma 12) the multiplicity of the zero is at least two. Since $B_{2} \subset C, d_{B_{2}} \leq d_{C}$ and $1-\left(4 \log d_{B_{2}}\right)^{-1} \leq 1-\left(4 \log d_{C}\right)^{-1} \leq \beta$, which contradicts Theorem $\left.2.6 \mathrm{~b}\right)$.

We have $L\left(\beta, \omega, K\left(\zeta_{q}\right) / E\right)=0$. By Proposition 2.4, $\omega$ is a real quadratic character. Since $\omega^{2}$ is a principal character, $\omega$ must evaluate to \pm 1 on all elements $g \in G=\operatorname{Gal}\left(K\left(\zeta_{q}\right) / E\right)$. Let $H<G$ consisting of all $g \in G$ such that $\omega(g)=1$, so $G / H \cong \mathbb{Z} / 2 \mathbb{Z}$. Let $N \subset K\left(\zeta_{q}\right)$ be the fixed field of $H$. By the Galois correspondence, $[N: E]=2$. By construction, $L(\beta, \omega, N / E)=0$. Since $[N: E]=2$, if $\sigma_{\mathfrak{p}}$ is the identity, $\omega\left(\sigma_{\mathfrak{p}}\right)=1$ and otherwise, $\omega\left(\sigma_{\mathfrak{p}}\right)=-1$.

The splitting type of the non-ramified prime ideal $\mathfrak{p}$ in $E$ is easily determined by the Frobenius elements $\sigma_{\mathfrak{p}}$. Let $\mathfrak{q}$ be a prime ideal of $N$ lying over $\mathfrak{p}$. The unramified prime ideal $\mathfrak{p}$ splits exactly when $N_{N / \mathbb{Q}} \mathfrak{q}=N_{E / \mathbb{Q}} \mathfrak{p}$, and is inert when $N_{N / \mathbb{Q}} \mathfrak{q}=N_{E / \mathbb{Q}} \mathfrak{p}^{2}$. Therefore, since $\sigma_{\mathfrak{p}}$ is defined as $\sigma_{\mathfrak{p}}(x)=x^{N_{E / \mathbb{Q}}}(\bmod \mathfrak{q})$, we see that $\sigma_{\mathfrak{p}}$ is the identity exactly when $\mathfrak{p}$ is split in $N$ and $\sigma_{\mathfrak{p}}$ is not the identity when $\mathfrak{p}$ is inert.

The unramified Euler product for $L(s, \omega, N / E$ ) (indicated with a subscript $u$ ) is

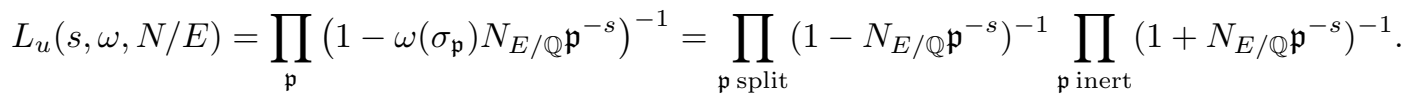

The unramified Euler product for $\zeta_{N}(s)$ is

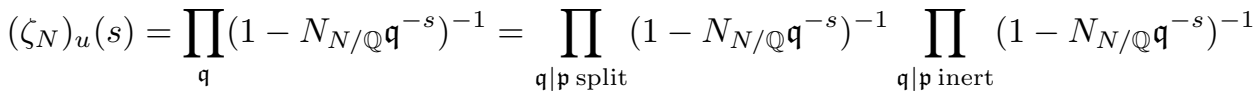

$$
\begin{aligned}
& =\prod_{\mathfrak{p} \text { split }}\left(1-N_{E / \mathbb{Q}} \mathfrak{p}^{-s}\right)^{-1} \prod_{\mathfrak{p} \text { inert }}\left(1-N_{E / \mathbb{Q}} \mathfrak{p}^{-2 s}\right)^{-1} \\
& =L_{u}(s, \omega, N / E) \prod_{\mathfrak{p} \text { inert }}\left(1-N_{E / \mathbb{Q}} \mathfrak{p}^{-s}\right)^{-1} \text {. }
\end{aligned}
$$


Therefore, $L(s, \omega, N / E)$ divides $\zeta_{N}(s)$ and $\beta$ is a zero of $\zeta_{N}$ as well. Similarly, by Aramata Brauer [1,5] one can see that $\zeta_{N}(s)$ divides $\zeta_{K N}(s)$, so $\beta$ is a zero of $\zeta_{K N}$ as well.

We may also view $\omega$ as a (real) quadratic Hecke character of $E$. The next proposition is an analog of Siegel's theorem giving a bound for the possible real Siegel zero in Proposition 2.4, and is an adaptation of Stark's [22] method. The purpose of this proposition is to get a bound for $\beta$ which only depends on $K$ and E.

Proposition 2.8. Let $\epsilon>0$ be given, and let $\omega$ be a non-trivial irreducible character of $K\left(\zeta_{q}\right) / E$ such that $A(\omega) \ll A q^{n_{E}}$. There is a positive constant $C=C(\epsilon)$ so that if $\beta$ is a Siegel zero of $L\left(s, \omega, K\left(\zeta_{q}\right) / E\right)$ then

$$
\beta \leq \max \left\{1-\frac{C}{\left(d_{E}^{2} A\right)^{2 \epsilon}}, 1-\frac{1}{4\left(2 n_{E}\right) ! n_{K} \log \left(d_{E}^{2} A\right)}\right\} .
$$

Proof. We will assume that $1-\left(4\left(2 n_{E}\right) ! n_{K} \log \left(d_{E}^{2} A\right)\right)^{-1} \leq \beta<1$ and prove that $\beta \leq 1-C\left(d_{E}^{2} A\right)^{-2 \epsilon}$.

By the conductor discriminant formula (0.4.1) $d_{K N} \leq d_{N}^{n_{K}}$. Moreover, since $[N: E]=2, d_{N / E}=A$, the conductor of $\zeta_{E}$, (since the only irreducible character is the principal character) and $d_{N}=d_{E}^{2} A$. We conclude that $d_{K N} \leq\left(d_{E}^{2} A\right)^{n_{K}}$, removing the dependence on $N$.

Therefore, if $\beta \geq 1-\left(4\left(2 n_{E}\right) ! n_{K} \log \left(d_{E}^{2} A\right)\right)^{-1}$ then $\beta \geq 1-\left(4\left(2 n_{E}\right) ! \log d_{K N}\right)^{-1}$. Applying Lemma 2.5 to the fields $\mathbb{Q} \subset E \subset N \subset K N$, we conclude that there is a quadratic subfield $S$ of $K N$ with $\zeta_{S}(\beta)=0$. By the classical Siegel theorem (cf. [7] $\S 21$ ) there is a constant $C=C(\epsilon)>0$ so that $\beta \leq 1-C d_{S}^{-\epsilon}$. (The conductor discriminant formula implies that $d_{S}=d_{S / \mathbb{Q}}=\mathfrak{f}$, the Artin conductor, since $S$ is quadratic.) Since $d_{S}^{n_{K} / 2} \leq d_{K N}$ we conclude that $\beta \leq 1-C d_{K N}^{-2 \epsilon / n_{K}} \leq 1-C\left(d_{E}^{2} A\right)^{-2 \epsilon}$ using the above discriminant relations.

We will need to estimate the contribution from the zeros close to the real line, and so require the following corollary to Proposition 2.4 and Proposition 2.8.

Corollary 2.9. Let $\omega$ be a non-trivial irreducible character of $K\left(\zeta_{q}\right) / E$ such that $A(\omega) \ll A q^{n_{E}}$. If $q \leq$ $(\log x)^{\gamma}$

$$
\sum_{|\rho|<\frac{1}{2}} \frac{1}{|\rho|} \ll(\log \log x)^{2}
$$

where the sum is over all non-trivial zeros of $L(s, \omega)$.

Proof. By [13] Lemma 5.4 the number of zeros in the region $|\rho|<\frac{1}{2}$ is $O\left(\log A(\omega)+n_{E} \log 2\right)$. Since $A(\omega) \ll A q^{n_{E}}$ and $q \leq(\log x)^{\gamma}$, the number of zeros is $O(\log \log x)$, where the implied constant depends only on $K, E$ and $\gamma$. By Proposition 2.4 and the functional equation for $L(s, \omega)$, there is at most one zero, $1-\beta$ in the region defined by $\operatorname{Re}(s) \leq C / \mathcal{L}(t)$ and by Proposition $2.8,1 /|1-\beta|$ is absolutely bounded. Therefore the summation is bounded by the number of zeros in the region $|\rho|<\frac{1}{2}$ multiplied by the maximum of $|\rho|^{-1}$ over the zeros in the region. It suffices to show that $|\rho|^{-1} \ll \log \log x$ for $|\rho| \leq \frac{1}{2}$ and $\operatorname{Re}(\rho)>C / \mathcal{L}(\operatorname{Im}(\rho))$.

For such a $\rho,|\rho|>|s|$ where $s=\sigma+i t$ is on the curve $C / \mathcal{L}(t)$ when $0 \leq t \leq \frac{1}{2}$. The modulus $|s| \geq \max \{t, C / \mathcal{L}(t)\}$ where $0 \leq t \leq \frac{1}{2}$. As $\mathcal{L}(t)=\frac{1}{2} \log A(\omega)+n_{E} \log (|t|+2)$, we conclude that when $0 \leq t \leq \frac{1}{2}$, there is a positive constant $D$ depending on $K, E$ and $\gamma$ so that $\mathcal{L}(t) \leq D \log \log x$. Therefore, $|\rho|>|s| \geq C / \mathcal{L}(t) \geq C /(D \log \log x)$ and so $|\rho|^{-1} \ll \log \log x$.

\subsection{Contour Integration}

We closely follow the proof of Theorem 7.1 in [13]. We sketch the ideas here as we need a slight modification of their work. Note that we are assuming that $\omega$ is an irreducible non-trivial character of $\operatorname{Gal}\left(K\left(\zeta_{q}\right) / E\right)$ and will leave off any terms which are only present when $\omega$ is the trivial character. 
We will first obtain estimates by contour integration. Recall that from (0.3.2)

$$
\tilde{\psi}(x, \omega)=\frac{1}{2 \pi i} \int_{(2)}-\frac{L^{\prime}}{L}(s, \omega) \frac{x^{s}}{s} d s .
$$

This differs from a truncated inverse Mellin transform

$$
I(T)=\frac{1}{2 \pi i} \int_{\sigma_{0}-i T}^{\sigma_{0}+i T}-\frac{L^{\prime}}{L}(s, \omega) \frac{x^{s}}{s} d s
$$

for $\sigma_{0}>1$ by a sufficiently small error term, $E_{1}$ for $1 \leq T \leq x$. We will let $\sigma_{0}=1+(\log x)^{-1}$. This truncated integral can be evaluated by contour integration and Cauchy's theorem. Specifically, we evaluate the contour integral

$$
I(B)=\frac{1}{2 \pi i} \int_{B}-\frac{L^{\prime}}{L}(s, \omega) \frac{x^{s}}{s} d s
$$

where $B$ is the positively oriented box with vertices at $\sigma_{0} \pm i T$, and $-U \pm i T$ where $U=j+\frac{1}{2}$ for some non-negative integer $j$. (We eventually let $U \rightarrow \infty$.) As in [13], we estimate

$$
I(T)-I(B)=E_{2} .
$$

By Cauchy's theorem $I(B)$ equals the sum of the residues of the integrand at poles inside $B$. Therefore, the main terms of $\tilde{\psi}(x, \omega)$ correspond to the residues of $L^{\prime} / L(s, \omega)$ inside $B$. Since $\omega$ is not the trivial character, $L(s, \omega)$ is analytic in $B$. As such, the main terms come from the zeros of $L$ in $B$. Let $S_{1}$ denote the contribution of the non-trivial zeros and $S_{2}$ the contribution of the trivial zeros. We have

$$
|\tilde{\psi}(x, \omega)|=I(T)+E_{1}=I(B)+E_{1}+E_{2}=S_{1}+S_{2}+E_{1}+E_{2} .
$$

The estimate of $E_{1}$ is very similar to the results in $\S 3$ of [13]. Therefore we only sketch the proof of this estimate. We use the following, which is Lemma 3.1 in [13].

Lemma 2.10. Let $y>0, \sigma>0$, and $T>0$ and let $I=\frac{1}{2 \pi i} \int_{\sigma-i T}^{\sigma+i T} \frac{y^{s}}{s} d s$. Then

1. $|I-1| \leq y^{\sigma} \min \left\{1, T^{-1}|\log y|^{-1}\right\}$ if $y>1$.

2. $\left|I-\frac{1}{2}\right| \leq \sigma T^{-1}$ if $y=1$.

3. $|I| \leq y^{\sigma} \min \left\{1, T^{-1}|\log y|^{-1}\right\}$ if $y<1$.

The estimates of $E_{1}, E_{2}, S_{1}$, and $S_{2}$ are summarized in the following, which is the main result of this section.

Proposition 2.11. Let $\omega$ be a non-trivial irreducible character of $K\left(\zeta_{q}\right) / E$ such that $A(\omega) \ll A q^{n_{E}}$. If $q \leq(\log x)^{\gamma}$ then for $1 \leq T \leq x$

$$
\tilde{\psi}(x, \omega)-\sum_{\rho} \frac{x^{\rho}}{\rho}-\sum_{\substack{\rho \\|\rho|<\frac{1}{2}}} \frac{1}{\rho} \ll \frac{n_{E}\|\omega\| x(\log x)^{2}}{T}
$$

where the summations are over the non-trivial zeros, $\rho$, of $L(s, \omega)$, and with $G=\operatorname{Gal}\left(K\left(\zeta_{q}\right) / E\right),\|\omega\|=$ $\max _{g \in G}|\omega(g)|$.

Proof. Using the definition of $\tilde{\psi}$ from (0.6.1) and writing out the summation for $-L^{\prime} / L$ as in (0.3.2) we can write the error $-E_{1}=I(T)-\tilde{\psi}(x, \omega)$ as

$$
\frac{1}{2 \pi i} \int_{\sigma_{0}-i T}^{\sigma_{0}+i T}\left(\sum_{\mathfrak{p}, m}(\log N \mathfrak{p}) \omega\left(\sigma_{\mathfrak{p}}^{m}\right)(N \mathfrak{p})^{-m s} \frac{x^{s}}{s} d s\right)-\sum_{N \mathfrak{p}^{m} \leq x}(\log N \mathfrak{p}) \omega\left(\sigma_{\mathfrak{p}}^{m}\right) .
$$


Switching the summation and integration, and grouping the three sums for $N \mathfrak{p}^{m}<x, N \mathfrak{p}^{m}=x$, and $N \mathfrak{p}^{m}>x$, allows us to use Lemma 2.10 and conclude that this error is bounded by the sum of the contribution of $N \mathfrak{p}^{m} \neq x$ and the contribution of $N \mathfrak{p}^{m}=x$. Specifically, it is bounded by

$$
\sum_{N \mathfrak{p}^{m} \neq x}(\log N \mathfrak{p}) \omega\left(\sigma \mathfrak{p}^{m}\right)\left(\frac{x}{N \mathfrak{p}^{m}}\right)^{\sigma_{0}} \min \left\{1, T^{-1}\left|\log \frac{x}{N \mathfrak{p}^{m}}\right|^{-1}\right\}+\sum_{N \mathfrak{p}^{m}=x}(\log N \mathfrak{p}) \omega\left(\sigma \mathfrak{p}^{m}\right)\left(\sigma_{0} T^{-1}-\frac{1}{2}\right) .
$$

The second summation is over at most $n_{E}$ elements $\mathfrak{p}^{m}$, so the sum is $O\left(n_{E}\|\omega\| \log x\left(\sigma_{0} T^{-1}-\frac{1}{2}\right)\right)$. With $\sigma_{0}=1+(\log x)^{-1}$ this term is $O\left(n_{E}\|\omega\|(\log x)\right)$ since $1 \leq T \leq x$. Substituting $\sigma_{0}=1+(\log x)^{-1}$ into the first term, by $[13](\S 3$ pages $425-428)$ we conclude that it is $O\left(\|\omega\| n_{E} \log x\left(1+x T^{-1} \log x\right)\right)$ for $x \geq 2$, and $T \geq 1$. Since $T \leq x$, we conclude that

$$
E_{1} \ll n_{E}\|\omega\| x T^{-1}(\log x)^{2} .
$$

To estimate $E_{2}$, we estimate the integral $I(B)$ over the other three sides of the box, $B$. To do so, we will assume that $T$ does not coincide with any ordinate zero of $L(s, \omega)$. The integral $I(B)$ defined on the box with vertices $\sigma_{0} \pm T$ and $-U \pm T$. We choose $U=j+\frac{1}{2}$ for some non-negative integer $j$. We are in the exact situation as [13] $\S 6$. From [13] (page 446 equation (6.13))

$$
E_{2} \ll x(\log x) T^{-1}\left(\log A(\omega)+n_{E} \log T\right)+T U^{-1} x^{-U}\left(\log A(\omega)+n_{E} \log (T+U)\right) .
$$

As $U \rightarrow \infty$,

$$
E_{2} \ll x(\log x) T^{-1}\left(\log A(\omega)+n_{E} \log T\right) .
$$

Therefore, as $U \rightarrow \infty$, since $T \leq x$

$$
E_{1}+E_{2} \ll x(\log x) T^{-1}\left(\log A(\omega)+\|\omega\| n_{E} \log x\right) .
$$

It now suffices to estimate $S_{1}$ and $S_{2}$. By Cauchy's theorem, $I(B)$ equals the sum of the residues of the integrand at the poles of $L^{\prime} / L(s, \omega)$ inside $B$. Since $\omega$ is not the trivial character (or a principal character, as $\omega$ is irreducible) the only poles are the first order poles, which have residue +1 , at the non-trivial zeros of $L(s, \omega)$ and the trivial zeros of $L(s, \omega)$. These are dealt with separately in the terms $S_{1}$ and $S_{2}$, respectively.

The term $S_{2}$ is the contribution from the trivial zeros. These correspond to the first order poles of $L^{\prime} / L$ at $s=-(2 m-1)$ for $m=1,2, \ldots$ with residue $a(\omega)$ and at $s=-2 m$ for $m=0,1, \ldots$ with residue $b(\omega)$. (See [13] page 442 equation (6.7).) The residues corresponding to the zeros contribute

$$
-b(\omega) \sum_{m=1}^{\left[\frac{U+1}{2}\right]} \frac{x^{-(2 m-1)}}{2 m-1}-a(\omega) \sum_{m=1}^{\left[\frac{U}{2}\right]} \frac{x^{-2 m}}{2 m} .
$$

Taking the limit as $U \rightarrow \infty$ this equals $\frac{1}{2}(a(\omega)+b(\omega)) \log \left(1-x^{-1}\right)+\frac{1}{2}(b(\omega)-a(\omega)) \log \left(1+x^{-1}\right)$. We can simplify this, since (see $\S 0.4) a(\omega)+b(\omega)=n_{E}$. The residue at $s=0$ contributes (see [13] pages 447-448) $r(\omega)+a(\omega) \log x$ where

$$
r(\omega)=B(\omega)-\frac{1}{2} \log A(\omega)+\frac{1}{2} n_{E} \log \pi-b(\omega) \frac{\Gamma^{\prime}}{\Gamma}\left(\frac{1}{2}\right)-\frac{1}{2} a(\omega) \frac{\Gamma^{\prime}}{\Gamma}(1)
$$

with $A(\omega)$ (the conductor) and $B(\omega)$ as in (0.7.2). Putting these together, the contribution of the trivial zeros is

$$
S_{2}=r(\omega)+a(\omega) \log x+\frac{1}{2} n_{E} \log \left(1-x^{-1}\right)+\frac{1}{2}(b(\omega)-a(\omega)) \log \left(1+x^{-1}\right) .
$$

From [13] (Proof of Theorem 7.1, using (5.5) and (5.4)) we see that

$$
r(\omega)-\sum_{\substack{\rho \\|\rho|<\frac{1}{2}}} \frac{1}{\rho} \ll \log A(\omega)+n_{E}
$$


The term $S_{2}$ is the contribution from the non-trivial zeros. Each non-trivial zero, $\rho$, has residue +1 and therefore the contribution is $\sum_{\rho} x^{\rho} \rho^{-1}$. Since $A(\omega) \ll A q^{d}$ and $q \leq(\log x)^{\gamma}$, by ( $\left.\dagger\right)$ and (††)

$$
S_{1}+S_{2} \ll \sum_{\rho} \frac{x^{\rho}}{\rho}+\sum_{|\rho|<\frac{1}{2}} \frac{1}{\rho}+\log A(\omega)+n_{E}(\log x) .
$$

Hence, $\tilde{\psi}(x, \omega) \ll S_{1}+S_{2}+E_{1}+E_{2}$ can be rewritten as

$$
\tilde{\psi}(x, \omega)-\sum_{\rho} \frac{x^{\rho}}{\rho}-\sum_{\substack{\rho \\|\rho|<\frac{1}{2}}} \frac{1}{\rho} \ll E .
$$

Since $T \leq x$, by $(*)$ and $(* *)$

$$
E=x(\log x) T^{-1}\left(\log A(\omega)+\|\omega\| n_{E} \log x\right)+\log A(\omega)+n_{E}(\log x) \ll n_{E}\|\omega\| x(\log x)^{2} T^{-1} .
$$

As stated, $T$ cannot equal the ordinate of any non-trivial zero. If $T$ does, as discussed in [13] (page 451 proof of Theorem 7.1) we can extend this estimate to such $T$ with an error term absorbed in our term.

\subsection{Estimates of zeros of $L(s, \omega)$}

This section is devoted to the proof of Proposition 2.2. From Proposition 2.11, we have

$$
\tilde{\psi}(x, \omega)-\sum_{\rho} \frac{x^{\rho}}{\rho}-\sum_{\substack{\rho \\|\rho|<\frac{1}{2}}} \frac{1}{\rho} \ll \frac{n_{E}\|\omega\| x(\log x)^{2}}{T}
$$

where the sums are over non-trivial zeros $\rho$ of $L(s, \omega), 2 \leq T \leq x$ and the first sum is over all $\rho$ such that $|\operatorname{Im}(\rho)|<T$.

Let

$$
T=\exp \left(\frac{1}{n_{E}}\left((\log x)^{\frac{1}{2}}-\frac{1}{2} \log A(\omega)\right)\right)-2,
$$

so that $\mathcal{L}(T)=(\log x)^{\frac{1}{2}}$. Since $A(\omega) \ll A q^{n_{E}}, q \leq(\log x)^{\gamma}$ and $T \leq x$, the error term, $n_{E}\|\omega\| x(\log x)^{2} T^{-1}$, satisfies the desired bound when $c<1 / n_{E}$.

Let $N(t, \omega)$ be the number of zeros of $L(s, \omega)$ in the region defined by $|t-\operatorname{Im}(\rho)| \leq 1$ and $0<\operatorname{Re}(\rho)<1$. The second summand, $\sum_{|\rho|<\frac{1}{2}}|\rho|^{-1} \ll(\log \log x)^{\frac{1}{2}}$, by Corollary 2.9 .

It remains to estimate

$$
\sum_{|\operatorname{Im}(\rho)|<T}\left|\frac{x^{\rho}}{\rho}\right|=\sum_{0 \leq|\operatorname{Im}(\rho)|<2}\left|\frac{x^{\rho}}{\rho}\right|+\sum_{2 \leq|\operatorname{Im}(\rho)|<T}\left|\frac{x^{\rho}}{\rho}\right| .
$$

The summation over $2 \leq|\operatorname{Im}(\rho)|<T$, can be treated as follows.

$$
\sum_{2 \leq|\operatorname{Im}(\rho)|<T} \frac{1}{|\rho|} \ll \sum_{2 \leq t<T} N(t, \omega) \max _{|t-\operatorname{Im}(\rho)| \leq 1} \frac{1}{|\rho|} \ll \sum_{2 \leq t<T} \frac{N(t, \omega)}{t} \ll \log T\left(\log A(\omega)+n_{E} \log T\right),
$$

by the standard estimate for $N(t, \omega)$ (as in Lemma 5.4 of [13] or p. 267 of [17]). Therefore, the second term has the bound

$$
\sum_{2 \leq|\operatorname{Im}(\rho)|<T}\left|\frac{x^{\rho}}{\rho}\right| \ll x^{g} \log x\left(\log A(\omega)+n_{E} \log x\right)
$$

where $g$ is the largest possible $|\operatorname{Re}(\rho)|$ in the range. By Proposition 2.4 and the symmetry of $L(s, \omega)$ about the $1 / 2$ line, there is a positive absolute constant $C$ so that these zeros are bounded by $1-C / \mathcal{L}(T)$, so 
$g \leq 1-C / \mathcal{L}(T)$. Choosing $T$ as above, $\mathcal{L}(T)=(\log x)^{\frac{1}{2}}$. Therefore, $x^{-C / \mathcal{L}(T)}=\exp \left(-C(\log x)^{\frac{1}{2}}\right)$ and the contribution of this portion of the sum is $x \log x\left(\log A(\omega)+n_{E} \log x\right) \exp \left(-C(\log x)^{\frac{1}{2}}\right)$. This satisfies the desired bound if $c<C$.

By Proposition 2.4 there is one possible Siegel zero $s=\sigma+i t$ in the region defined by $1-C / \mathcal{L}(t) \leq \sigma \leq 1$. Let $\beta$ denote this zero, if it exists. By symmetry, there is one possible Siegel zero, $1-\beta$ in the region defined by $0 \leq 1-\sigma \leq C / \mathcal{L}(t)$. First, we will bound the sum for the non-Siegel zeros, $\rho$, in the region $0 \leq|\operatorname{Im}(\rho)| \leq 2$. By Proposition 2.4 these $\rho$ satisfy

$$
|\operatorname{Re}(\rho)|<1-\frac{C}{\mathcal{L}(\operatorname{Im}(\rho))}<1-\frac{C}{\mathcal{L}(2)}=1-\frac{C}{\frac{1}{2} \log A(\omega)+n_{E} \log 4} .
$$

Therefore, for such a zero

$$
\left|\frac{x^{\rho}}{\rho}\right| \ll x x^{-C\left(\frac{1}{2} \log A(\omega)+n_{E} \log 4\right)^{-1}}|\rho|^{-1}
$$

where

$$
|\rho|^{-1} \leq \frac{\mathcal{L}(\operatorname{Im}(\rho))}{C} \leq \frac{\mathcal{L}(2)}{C}=\left(\frac{1}{2} \log A(\omega)+n_{E} \log 4\right) C^{-1} \ll \log A(\omega)
$$

where the implied constant depends only on $K$ and $C$. There are only finitely many such zeros in this region, bounded by $N(1, \omega)+N(2, \omega) \ll n_{E}+\log A(\omega)$ by [13] Lemma 5.4. Therefore the total summation over the non-Siegel zeros is bounded by

$$
\left(n_{E}+\log A(\omega)\right) \log A(\omega) x x^{-C\left(\frac{1}{2} \log A(\omega)+n_{E} \log 4\right)^{-1}} .
$$

A straightforward computation shows that this satisfies the desired bound if $c<C$.

It remains to show the bound for the possible Siegel zeros. That is, it suffices to bound $x^{\beta} / \beta$ and $x^{1-\beta} /(1-\beta)$ where $\beta$ is the (real) Siegel zero in the range $\frac{1}{2} \leq \beta<1$. These are both majorized by $x^{\beta} /(1-\beta)$. By Proposition 2.8

$$
\beta \leq \max \left\{1-\frac{C(\epsilon)}{\left(d_{E} A\right)^{2 \epsilon}}, 1-\frac{1}{4\left(2 n_{E}\right) ! n_{K} \log \left(d_{K}^{2} A\right)}\right\}=1-D
$$

for some constant $D=D(\epsilon, E, A, K)$. Therefore,

$$
\frac{x^{\beta}}{1-\beta} \ll x x^{-D} D^{-1}
$$

It is elementary to verify that $x^{-D} \ll \exp \left(-c(\log x)^{\frac{1}{2}}\right)$ if $c<C$.

\section{The Terminal Range}

Proposition 2.1 allows us to estimate the average for $Q \leq(\log x)^{\gamma}$. The following proposition estimates the portion of the range greater than $(\log x)^{\gamma}$. We will let $d=n_{E}$ as defined in Definition 1.1.

Proposition 3.1 (Terminal Range). Let $K / E$ be an abelian Galois extension, and let $D$ and $\gamma$ be any positive constants with $\gamma>4 d+3+D$. For $\epsilon>0,(\log x)^{\gamma} \leq Q \leq \min \left\{x^{\frac{1}{2}-\epsilon}, x^{\frac{1}{d-2}-\epsilon}\right\}$ and $k>\max \left\{2, \frac{d}{2}\left(1+\frac{d}{\epsilon}\right)\right\}$,

$$
\frac{1}{Q} \sum_{q \leq Q} \sum_{\omega}^{*} \max _{y \leq x}\left|\tilde{\psi}_{k}\left(y, \omega, K\left(\zeta_{q}\right) / E\right)\right| \ll \frac{x}{(\log x)^{D}}
$$

where the inner summation is over all irreducible, non-trivial characters $\omega$ of $K\left(\zeta_{q}\right) / E$ such that $A(\omega) \ll A q^{d}$. 
We will prove this using an adaptation of a method of Gallagher[10] as modified by Bombieri ([4] §7.2). First, we establish some notation. As the characters $\omega$ in Proposition 3.1 are abelian, we will now adopt the notation of Dirichlet characters. We write

$$
\begin{aligned}
L(s, \omega) & =\sum_{n=1}^{\infty} a_{n} \omega(n) n^{-s} \\
\frac{1}{L}(s, \omega) & =\sum_{n=1}^{\infty} b_{n} \omega(n) n^{-s} \\
-\frac{L^{\prime}}{L}(s, \omega) & =\sum_{n=1}^{\infty} c_{n} \Lambda(n) \omega(n) n^{-s} .
\end{aligned}
$$

Letting $0<z \leq x$ be a parameter to be specified later, we start with the identity $[10,3]$

$$
-\frac{L^{\prime}}{L}(s, \omega)=G(1-L M)+F(1-L M)-L^{\prime} M
$$

where $L=L(s, \omega), L^{\prime}=L^{\prime}(s, \omega)$ and

$$
\begin{aligned}
M=M_{z}(s, \omega) & =\sum_{n \leq z} b_{n} \omega(n) n^{-s} \\
F=F_{z}(s, \omega) & =\sum_{n \leq z} c_{n} \Lambda(n) \omega(n) n^{-s} \\
G=G_{z}(s, \omega) & =\sum_{n>z} c_{n} \Lambda(n) \omega(n) n^{-s} .
\end{aligned}
$$

From (0.6.4)

$$
\tilde{\psi}_{k}(x, \omega)=\frac{1}{2 \pi i} \int_{(2)}-\frac{L^{\prime}}{L}(s, \omega) \frac{x^{s}}{s^{k+1}} d s
$$

and in fact, we can move the line of integration to any $C>1$. Integrating (3.0.1),

$$
\tilde{\psi}_{k}(x, \omega)=\frac{1}{2 \pi i} \int_{(C)} G(1-L M) \frac{x^{s}}{s^{k+1}} d s+\frac{1}{2 \pi i} \int_{(C)} F(1-L M) \frac{x^{s}}{s^{k+1}} d s-\frac{1}{2 \pi i} \int_{(C)} L^{\prime} M \frac{x^{s}}{s^{k+1}} d s .
$$

Since $F$ and $M$ are Dirichlet polynomials and $L, L^{\prime}$ are analytic for all $s$, we can move the line of integration in the second and third terms of the above expression to $\Re(s)=1 / 2$.

Therefore,

$$
\begin{aligned}
\sum_{q \leq Q} \sum_{\omega}^{*} \max _{y \leq x}\left|\tilde{\psi}_{k}(y, \omega)\right| & \ll \sum_{q \leq Q} \sum_{\omega}^{*} \max _{y \leq x} \int_{(C)}|G(1-L M)| \frac{\left|y^{s}\right|}{\left|s^{k+1}\right|}|d s| \\
& +\sum_{q \leq Q} \sum_{\omega}^{*} \max _{y \leq x} \int_{(1 / 2)}\left(|F(1-L M)|+\left|L^{\prime} M\right|\right) \frac{\left|y^{s}\right|}{\left|s^{k+1}\right|}|d s| .
\end{aligned}
$$

Repeatedly using the inequality $2|a b| \leq|a|^{2}+|b|^{2}$,

$$
\begin{aligned}
\sum_{q \leq Q} \sum_{\omega}{ }^{*} \max _{y \leq x}\left|\tilde{\psi}_{k}(y, \omega)\right| & \ll \sum_{q \leq Q} \sum_{\omega}{ }^{*} \max _{y \leq x} \int_{(C)}\left(|G|^{2}+|1-L M|^{2}\right) \frac{\left|y^{s}\right|}{\left|s^{k+1}\right|}|d s| \\
& +\sum_{q \leq Q} \sum_{\omega}{ }^{*} \max _{y \leq x} \int_{(1 / 2)}\left(1+|F|^{2}+|M|^{2}+|F M|^{2}+|L|^{2}+\left|L^{\prime}\right|^{2}\right) \frac{\left|y^{s}\right|}{\left|s^{k+1}\right|}|d s| .
\end{aligned}
$$

For the first integral, we move the line of integration to $C=1+(\log x)^{-1}$ so that $\left|y^{s}\right| \ll y x^{1 / \log x} \ll y$. For the second integral, as $F, M$ are Dirichlet polynomials and $L$ and $L^{\prime}$ are analytic for all $s$ we can move the line of integration onto the critical line, so $\left|y^{s}\right| \leq y^{\frac{1}{2}}$. We obtain the bound

$$
\sum_{q \leq Q} \sum_{\omega}^{*} \max _{y \leq x}\left|\tilde{\psi}_{k}(y, \omega)\right| \ll x S_{1}+x S_{2}+x^{\frac{1}{2}} S_{3}+x^{\frac{1}{2}} S_{4},
$$


where

$$
\begin{aligned}
S_{1} & =\sum_{q \leq Q} \sum_{\omega}^{*} \int_{(C)}|G|^{2} \frac{|d s|}{|s|^{k+1}} \\
S_{2} & =\sum_{q \leq Q} \sum_{\omega}^{*} \int_{(C)}|1-L M|^{2} \frac{|d s|}{|s|^{k+1}} \\
S_{3} & =\sum_{q \leq Q} \sum_{\omega}^{*} \int_{\left(\frac{1}{2}\right)}\left(1+|F|^{2}+|M|^{2}+|F M|^{2}\right) \frac{|d s|}{|s|^{k+1}} \\
S_{4} & =\sum_{q \leq Q} \sum_{\omega}^{*} \int_{\left(\frac{1}{2}\right)}\left(|L|^{2}+\left|L^{\prime}\right|^{2}\right) \frac{|d s|}{|s|^{k+1}} .
\end{aligned}
$$

In $\S 3.1$, we estimate $S_{2}, S_{2}$ and $S_{3}$ using Gallagher's large sieve identity. After making some initial estimates in $\S 3.2$ we use mean value estimates to handle $S_{4}$ in $\S 3.3$. These estimates are summarized in Propositions 3.5 and 3.10, respectively. In $\S 3.4$ these estimates are combined to prove Proposition 3.1.

\subsection{Gallagher's Large Sieve Inequality}

We use the following form of the large sieve ([4] Théorème 10).

Theorem 3.2. Let $\sum_{n=1}^{\infty}\left|A_{n}\right|<\infty$ and $T \geq 1$. Then

$$
\sum_{q \leq Q} \sum_{\omega}^{*} \int_{-T}^{T}\left|\sum_{n=1}^{\infty} A_{n} \omega(n) n^{i t}\right|^{2} d t \ll \sum_{n=1}^{\infty}\left|A_{n}\right|^{2}\left(n+Q^{2} T\right) .
$$

We will often use Theorem 3.2 in the following form.

Corollary 3.3. Let $\sum_{n=1}^{\infty}\left|A_{n}\right|<\infty$ and $C \neq 0$. Then for $k \geq 2$ and $T \geq 1$ the sums

$$
\sum_{q \leq Q} \sum_{\omega}^{*} \int_{-T}^{T}\left|\sum_{n=1}^{\infty} \frac{A_{n} \omega(n)}{n^{C+i t}}\right|^{2} \frac{d t}{(C+|t|)^{k+1}}, \quad \text { and } \quad \sum_{q \leq Q} \sum_{\omega}^{*} \int_{(C)}\left|\sum_{n=1}^{\infty} \frac{A_{n} \omega(n)}{n^{s}}\right|^{2} \frac{|d s|}{|s|^{k+1}}
$$

are both $O\left(\left(|C|^{-k-1}+1\right) \sum_{n=1}^{\infty}\left|A_{n}\right|^{2} n^{-2 C}\left(n+Q^{2}\right)\right)$.

Proof. We rewrite the second integral as the real valued integral

$$
\sum_{q \leq Q} \sum_{\omega}^{*} \int_{-\infty}^{\infty}\left|\sum_{n=1}^{\infty} B_{n} \omega(n) n^{i t}\right|^{2}|C-i t|^{-k-1} d t
$$

where $B_{n}=A_{n} n^{-C}$. We evaluate this over the interval $[-1,1]$, and proceed radially out, over the intevals $[-(j+1),-j]$ and $[j, j+1]$ where $1<j \leq T$, and then take the limit as $T \rightarrow \infty$. By Theorem 3.2,

$$
\sum_{q \leq Q} \sum_{\omega}^{*} \int_{-1}^{1}\left|\sum_{n=1}^{\infty} B_{n} \omega(n) n^{i t}\right|^{2}|C-i t|^{-k-1} d t \ll \sum_{n=1}^{\infty} \frac{\left|A_{n}\right|^{2}}{|C|^{k+1} n^{2 C}}\left(n+Q^{2}\right) .
$$

When $t \in[-(j+1),-j] \cup[j, j+1]$ the quantity $|C-i t| \geq j$ so the contribution of (3.1.1) when $t$ is restricted this range is bounded by

$j^{-k-1} \sum_{q \leq Q} \sum_{\omega} *\left(\int_{-(j+1)}^{-j}\left|\sum_{n=1}^{\infty} B_{n} \omega(n) n^{i t}\right|^{2} d t+\int_{j}^{j+1}\left|\sum_{n=1}^{\infty} B_{n} \omega(n) n^{i t}\right|^{2} d t\right) \ll \frac{1}{j^{k+1}} \sum_{n=1}^{\infty} \frac{\left|A_{n}\right|^{2}}{n^{2 C}}\left(n+(j+1) Q^{2}\right)$. 
The estimate of the truncated integral from $-T$ to $T$ is of the order

$$
\sum_{n=1}^{\infty} \frac{\left|A_{n}\right|^{2}}{|C|^{k+1} n^{2 C}}\left(n+Q^{2}\right)+\sum_{j=2}^{[T]} \frac{1}{j^{k+1}} \sum_{n=1}^{\infty} \frac{\left|A_{n}\right|^{2}}{n^{2 C}}\left(n+(j+1) Q^{2}\right)
$$

Summing over $j$, the second summation is of the order

$$
\sum_{n=1}^{\infty} \frac{\left|A_{n}\right|^{2}}{n^{2 C}}\left(\frac{T^{-k}+2^{-k}}{k} n+\frac{T^{-k+1}+2^{-k+1}}{1-k} Q^{2}\right)\left(|C|^{-k-1}+1\right) .
$$

Taking the limit as $T \rightarrow \infty$ and choosing $k \geq 2$ to ensure convergence, we find (3.1.2) is of the order

$$
\sum_{n=1}^{\infty} \frac{\left|A_{n}\right|^{2}}{n^{2 C}}\left(n+Q^{2}\right)\left(|C|^{-k-1}+1\right)
$$

We now estimate the coefficients $a_{n}, b_{n}$, and $c_{n}$.

Lemma 3.4. Let $\tau_{d}(n)$ be the number of ways of writing $n$ as a product of $d$ natural numbers. Let $a_{n}$ be the coefficients of $L(s, \omega), b_{n}$ the coefficients of $\frac{1}{L}(s, \omega)$, and $c_{n}$ the coefficients of $-\frac{L^{\prime}}{L}(s, \omega)$. Then $0 \leq\left|a_{n}\right| \leq d ! \tau_{d}(n),\left|b_{n}\right| \leq d ! \tau_{2}(n), \max _{r \mid n, r \leq z}\left|b_{r}\right| \leq d ! \tau_{2}(n)$, and $0 \leq\left|c_{n}\right| \leq\left|a_{n}\right| \tau_{2}(n)$.

Proof. For a rational prime $p$, if there are $l$ prime ideals in $E$ above $p$, label these prime ideals by positive integers up to $l$. Let 1 correspond to the last $d-l$ labels. Given an ordered product $n=n_{1} n_{2} \ldots n_{d}$ write the ideal corresponding to $n_{i}$ as follows. Factor $n_{i}$ into rational primes and take all ideals with the $i^{\text {th }}$ label which lie over each prime divisor of $n_{i}$. Since there are $d$ ! orderings, and $a_{n}$ is bounded by the number of ideals of norm $n, a_{n} \leq d ! \tau_{d}(n)$ where $\tau_{j}(n)$ is the number of ways of writing $n$ as an unordered product of $j$ positive integers.

For the coefficients $b_{n}$, we have the relation that $b_{1}=1$ and for $n>1$

$$
\sum_{n_{1} n_{2}=n} a_{n_{1}} b_{n_{2}}=0
$$

The coefficient $b_{n}$ is multiplicative, but not totally multiplicative. For any additive partition $\rho$ of $m$ given by $m_{1}+\cdots+m_{t}=m$ consider the product $a_{\rho}=a_{p^{m_{1}}} \ldots a_{p^{m_{t}}}$. Then, $-b_{p^{m}}=\sum_{\rho} \mu(\rho) a_{\rho}$ where the weight $\mu(\rho)$ is zero if $\rho$ has any repeated summands. Otherwise, it is 1 if the length of $\rho$ is odd and -1 if the length is even. If $p$ is a prime, $-b_{p}=a_{p}$ and and more generally, if $m<d=n_{E}, b_{p^{m}} \leq\left(\begin{array}{c}d \\ m\end{array}\right)$. If $m>d$ it follows that $b_{p^{m}}=0$. Therefore $\max _{r \mid n, r \leq z}\left|b_{r}\right| \leq 2 \tau_{2}(n) \max _{p^{m} \mid n}\left|b_{p^{m}}\right| \leq 2 \tau_{2}(n)\left(\begin{array}{c}d \\ \left\lfloor\frac{d}{2}\right\rfloor\end{array}\right) \leq d ! \tau_{2}(n)$, and also $\left|b_{n}\right| \leq d ! \tau_{2}(n)$.

For the coefficients $c_{n}$ we need only consider values of $n$ which are prime powers. From the relation

$$
a_{p^{M}}=\sum_{m=1}^{M} c_{p^{m}} a_{p^{M-m}}
$$

we deduce that

$$
c_{p^{m}}=\sum_{m_{1} m_{2}=m} m_{1}\left(p_{m_{1}}\right)^{m_{2}}
$$

where $p_{m_{1}}$ is the number of prime ideals of norm $p^{m_{1}}$. The estimate follows directly.

We remark that the precision of the inequalities for $a_{n}, b_{n}, c_{n}$ derived in the above lemma are not essential for our estimates below. What is essential is that they are bounded by divisor functions. One knows, for instance that

$$
\sum_{n=1}^{\infty} \frac{\tau_{d}(n)}{n^{s}}=\zeta(s)^{d}
$$


and standard methods of estimation (see for example, Exercise 4.4 .17 on p. 67 of [16]) lead to the bounds

$$
\sum_{n \leq x} \tau_{d}(n) \ll x(\log x)^{d-1}
$$

and

$$
\sum_{n \leq x} \tau_{d}(n)^{2} \ll x(\log x)^{d(d+1)},
$$

and these will be used repeatedly below as in [17].

We will now prove the following estimates for $S_{1}, S_{2}$ and $S_{3}$. We will estimate $S_{4}$ in several parts, in $\S 3.2$ and $\S 3.3$.

Proposition 3.5. For $k \geq 2, S_{1}=O\left((d !)^{2}(\log x)^{(d+2)^{2}}\left(1+\frac{Q^{2}}{z}\right)\right), S_{2}=O\left((d !)^{2}(\log x)^{(d+2)^{2}}\left(1+\frac{Q^{2}}{z}\right)\right)$ and $S_{3}=O\left((d !)^{4}(\log z)^{(d+3)^{2}}\left(z^{2}+Q^{2}\right)\right)$.

Proof. We begin with $S_{1}$. With $C=1+(\log x)^{-1}$,

$$
S_{1}=\sum_{q \leq Q} \sum_{\omega} * \int_{(C)}\left|\sum_{n>z} \frac{c_{n} \Lambda(n) \omega(n)}{|s|^{\frac{k+1}{2}} n^{s}}\right|^{2}|d s| .
$$

By Corollary 3.3, since $C>1$

$$
S_{1} \ll \sum_{n>z} \frac{c_{n}^{2} \Lambda(n)^{2}}{n^{2 C}}\left(n+Q^{2}\right) \ll(d !)^{2} \sum_{n>z} \frac{\tau_{d+1}(n)^{2}}{n^{2+2(\log x)^{-1}}}\left(n+Q^{2}\right) .
$$

Estimating the dominant integral,

$$
S_{1} \ll(d !)^{2}(\log x)^{(d+2)^{2}} z^{-2(\log x)^{-1}}\left(1+\frac{Q^{2}}{z}\right) \ll(d !)^{2}(\log x)^{(d+2)^{2}}\left(1+\frac{Q^{2}}{z}\right) .
$$

By definition

$$
S_{2}=\sum_{q \leq Q} \sum_{\omega}^{*} \int_{(C)}|1-L M|^{2} \frac{|d s|}{|s|^{k+1}}
$$

and

$$
1-L M=-\sum_{n>z} \sum_{\substack{r \mid n \\ r \leq z}} b_{r} \mu(r) a_{n / r} \omega(n) n^{-s}=: \sum_{n>z} t_{n} \omega(n) n^{-s} .
$$

By Lemma 3.4

$$
t_{n}=\left|\sum_{\substack{r \mid n \\ r \leq z}} b_{r} a_{n / r} \mu(r)\right| \leq d ! \tau_{d}(n) \sum_{\substack{r \mid n \\ r \leq z}}\left|b_{r}\right| \leq(d !)^{2} \tau_{d+1}(n) .
$$

By Corollary 3.3, since $C=1+(\log x)^{-1}$,

$$
S_{2} \ll \sum_{n>z} \frac{\left|t_{n}\right|^{2}}{n^{2+2(\log x)^{-1}}}\left(n+Q^{2}\right) \ll(d !)^{4} \sum_{n>z} \frac{\tau_{d+1}(n)^{2}}{n^{2+2(\log x)^{-1}}}\left(n+Q^{2}\right) \ll(d !)^{4}(\log x)^{(d+2)^{2}}\left(1+\frac{Q^{2}}{z}\right) .
$$

Now we estimate

$$
S_{3}=\sum_{q \leq Q} \sum_{\omega} * \int_{\left(\frac{1}{2}\right)}\left(1+|F|^{2}+|M|^{2}+|F M|^{2}\right) \frac{|d s|}{|s|^{k+1}}
$$

We take each summand in the integrand separately. To compute the first integral (with integrand 1), we write the integral as a real valued integral and compute it on intervals as in the proof of Corollary 3.3, proceeding radially out. This gives

$$
\sum_{q \leq Q} \sum_{\omega}^{*}\left(2^{k+2}+\sum_{k=2}^{\infty} j^{-(k+1)}\right) \ll \sum_{q \leq Q} \sum_{\omega}^{*} 1 \ll \sum_{q \leq Q} \phi(q) \ll Q^{2} .
$$


We use Corollary 3.3 for the rest of the terms. Since $F=\sum_{n \leq z} c_{n} \Lambda(n) \omega(n) n^{-s}$ and the integration is along the line $\left(\frac{1}{2}\right)$, the second term is of the order

$$
(d !)^{2} \sum_{n \leq z} \frac{\tau_{d+1}(n)^{2}}{n}\left(n+Q^{2}\right) \ll(d !)^{2}(\log z)^{(d+2)^{2}}\left(z+(\log z) Q^{2}\right) \ll(d !)^{2}(\log z)^{(d+3)^{2}}\left(z+Q^{2}\right) .
$$

Since $M=\sum_{n \leq z} b_{n} \omega(n) n^{-s}$, the third term is of the order

$$
(d !)^{2} \sum_{n \leq z} \frac{\tau_{2}(n)^{2}}{n}\left(n+Q^{2}\right) \ll(d !)^{2}(\log z)^{6}\left(z+Q^{2}\right) .
$$

Finally, since $F M=\sum_{\substack{n_{1}, n_{2} \leq z \\ n_{1} n_{2}=n}} \omega(n)\left(c_{n_{1}} \Lambda\left(n_{1}\right) b_{n_{2}}\right) n^{-s}$, the last term is of the order

$$
(d !)^{4} \sum_{n \leq z^{2}} \frac{\tau_{d+2}(n)^{2}}{n}\left(n+Q^{2}\right) \ll(d !)^{4}(\log z)^{(d+3)^{2}}\left(z^{2}+Q^{2}\right) .
$$

\subsection{Phragmén-Lindelöf Estimates}

Write $S_{4}=S_{4}^{\prime}+S_{4}^{\prime \prime}$ where

$$
S_{4}^{\prime}=\sum_{q \leq Q} \sum_{\omega}^{*} \int_{\left(\frac{1}{2}\right)} \frac{|L(s, \omega)|^{2}|d s|}{|s|^{k+1}} \text { and } \quad S_{4}^{\prime \prime}=\sum_{q \leq Q} \sum_{\omega}^{*} \int_{\left(\frac{1}{2}\right)} \frac{\left|L^{\prime}(s, \omega)\right|^{2}|d s|}{|s|^{k+1}} .
$$

Rewrite the integral over the line $\frac{1}{2}+i t$ as a real valued integral over $t$,

$$
S_{4}^{\prime}=\sum_{q \leq Q} \sum_{\omega}^{*} \int_{-\infty}^{\infty} \frac{\left|L\left(\frac{1}{2}+i t, \omega\right)\right|^{2} d t}{\left(\frac{1}{2}+|t|\right)^{k+1}} \quad \text { and } \quad S_{4}^{\prime \prime}=\sum_{q \leq Q} \sum_{\omega}^{*} \int_{-\infty}^{\infty} \frac{\left|L^{\prime}\left(\frac{1}{2}+i t, \omega\right)\right|^{2} d t}{\left(\frac{1}{2}+|t|\right)^{k+1}} \text {. }
$$

In this section we will estimate the tail ends of the integral, and we estimate the portion of the integral over $[-T, T]$ in $\S 3.3$. First, we prove an estimate for $L$ and $L^{\prime}$.

Proposition 3.6. For $0 \leq \sigma \leq 1$, if $d \geq 2$ then

$$
|L(\sigma+i t, \omega)| \ll\left[A(\omega)(|t|+2)^{d}\right]^{\frac{1-\sigma}{2}}\left[\log \left(A(\omega)(|t|+2)^{d}\right)\right]^{d}
$$

and

$$
\left|L^{\prime}(\sigma+i t, \omega)\right| \ll d^{d}\left[A(\omega)(|t|+2)^{d}\right]^{\frac{1-\sigma}{2}}\left[\log \left(A(\omega)(|t|+2)^{d}\right)\right]^{d+1} .
$$

This follows from the following sharp form of the Phragmén-Lindelöf theorem by Rademacher ([20] Theorem 2).

Theorem 3.7 (Rademacher). Let $s=\sigma+i t$. Let $f(s)$ be a regular function on the vertical strip $c_{1} \leq \sigma \leq c_{2}$. For positive constants $\delta, C_{1}, C_{2}, \alpha_{1}$, and $\alpha_{2}$ and a constant $Q$ assume that

1. $|f(s)| \ll e^{|s|^{\delta}}$ on the strip $c_{1} \leq \sigma \leq c_{2}$.

2. $\left|f\left(c_{1}+i t\right)\right| \leq C_{1}\left|Q+c_{1}+i t\right|^{\alpha_{1}}$.

3. $\left|f\left(c_{2}+i t\right)\right| \leq C_{2}\left|Q+c_{2}+i t\right|^{\alpha_{2}}$. 
Then we have the following estimate for $f(s)$ in the strip $c_{1} \leq \sigma \leq c_{2}$

$$
|f(s)| \leq\left(C_{1}|Q+s|^{\alpha_{1}}\right)^{\frac{c_{2}-\sigma}{c_{2}-c_{1}}}\left(C_{2}|Q+s|^{\alpha_{2}}\right)^{\frac{\sigma-c_{1}}{c_{2}-c_{1}}} .
$$

Proof of Proposition 3.6. We begin with the estimate for $L(\sigma+i t)$. For $\sigma>0$ the Euler product for $L(s, \omega)$ can be written as $L(s, \omega)=\prod_{p} L_{p}(s, \omega)$ where $L_{p}$ is a polynomial in $p^{-s}$ of degree $m_{p} \leq d$ and the product is over all rational primes (see $(0.3 .1)$ ). For some $\left|\pi_{p, j}\right|=1$ we have

$$
L_{p}(s, \omega)=\prod_{j=1}^{m_{p}}\left(1-\frac{\pi_{p, j}}{p^{s}}\right)^{-1}
$$

Since $\left|1-\pi_{p, j} p^{-(1+\epsilon+i t)}\right| \geq\left|1-p^{-(1+\epsilon)}\right|$ we conclude that

$$
|L(1+\epsilon+i t, \omega)| \leq \zeta(1+\epsilon)^{d}
$$

where $\zeta(s)$ is the Riemann zeta function.

By the functional equation (0.4.3) for $L(s, \omega)$ evaluated at $s=-\epsilon+i t$,

$$
L(-\epsilon+i t, \omega)=\Theta(-\epsilon+i t, \omega) L(1+\epsilon-i t, \bar{\omega})
$$

and by the estimate for $\Theta$ in (0.4.5),

$$
L(-\epsilon+i t, \omega) \ll\left[A(\omega)(|t|+2)^{d}\right]^{\frac{1}{2}+\epsilon} L(1+\epsilon-i t, \bar{\omega}) .
$$

With $(*)$ we conclude that

$$
L(-\epsilon+i t, \omega) \ll\left[A(\omega)(|t|+2)^{d}\right]^{\frac{1}{2}+\epsilon} \zeta(1+\epsilon)^{d} .
$$

Let $c_{1}=-\epsilon, c_{2}=1+\epsilon, C_{1}=A(\omega)^{\frac{1}{2}+\epsilon} \zeta(1+\epsilon)^{d}, C_{2}=\zeta(1+\epsilon)^{d}, \alpha_{1}=d\left(\frac{1}{2}+\epsilon\right)$, and $\alpha_{2}=0$. Replacing $\left|Q+c_{1}+i t\right|$ with $(|t|+2)$ in Theorem 3.7, we have the estimate

$$
|L(\sigma+i t, \omega)| \leq \zeta(1+\epsilon)^{d}\left[A(\omega)(|t|+2)^{d}\right]^{\frac{1-\sigma+\epsilon}{2}}
$$

which is valid for $-\frac{1}{2} \leq-\epsilon \leq \sigma \leq 1+\epsilon \leq \frac{3}{2}$.

Setting $\epsilon=\left(\log \left[A(\omega)(|t|+2)^{d}\right]\right)^{-1}$ satisfies $0<\epsilon \leq \frac{1}{2}$ if $d \geq 2$. From the Laurent series expansion of $\zeta(s)$ at $s=1$ we see that $\zeta(1+\epsilon) \ll \epsilon^{-1}$. The estimate follows since $\left[A(\omega)(|t|+2)^{d}\right]^{\frac{1}{2}\left(\log \left[A(\omega)(|t|+2)^{d}\right]\right)^{-1}}$ is bounded.

For the $L^{\prime}$ estimate, we use the maximum modulus theorem. Since $L$ is holomorphic in the disk of radius $\epsilon$ about $s$, (Since $\sigma \leq 1+\epsilon$, the ball of radius $\epsilon$ about $s$ is contained in the strip $0<\sigma<1$ if $s$ is in this strip.)

$$
L^{\prime}(s, \omega) \leq \epsilon^{-1} M(\epsilon)
$$

where $M(\epsilon)$ is the maximum of $L(s, \omega)$ on a ball of radius $\epsilon$ about $s$. If $s$ is on the boundary, the estimate holds by continuity. Using the above $L$ bounds results in an additional multiplicative factor of $d^{d} \log \left(A(\omega)(|t|+2)^{d}\right)$.

We now prove the first portion of the estimate for $S_{4}^{\prime}$ and $S_{4}^{\prime \prime}$.

Lemma 3.8. For $0<\epsilon<\frac{1}{4}$, with $I=(-\infty,-T] \cup[T, \infty)$ for any $T \geq 1$,

$$
\sum_{q \leq Q} \sum_{\omega} * \int_{I} \frac{\left|L\left(\frac{1}{2}+i t, \omega\right)\right|^{2} d t}{\left(\frac{1}{2}+|t|\right)^{k+1}} \ll A^{\frac{1}{2}} Q^{\frac{d}{2}+2} \log \left(A Q^{d}\right)^{2 d} d^{2 d} \frac{(\log T)^{2 d}}{\left(k-\frac{d}{2}\right) T^{k-\frac{d}{2}}}
$$

and

$$
\sum_{q \leq Q} \sum_{\omega} * \int_{I} \frac{\left|L^{\prime}\left(\frac{1}{2}+i t, \omega\right)\right|^{2} d t}{\left(\frac{1}{2}+|t|\right)^{k+1}} \ll A^{\frac{1}{2}} Q^{\frac{d}{2}+2} \log \left(A Q^{d}\right)^{2 d+2} d^{2 d+1} \frac{(\log T)^{2 d+2}}{\left(k-\frac{d}{2}\right) T^{k-\frac{d}{2}}}
$$


Proof. The Phragmén-Lindelöf estimate in the form of Proposition 3.6 implies that

$$
\begin{aligned}
\int_{T}^{\infty} \frac{\left|L\left(\frac{1}{2}+i t, \omega\right)\right|^{2} d t}{\left(\frac{1}{2}+|t|\right)^{k+1}} & \ll \int_{T}^{\infty}\left[A(\omega)(|t|+2)^{d}\right]^{\frac{1}{2}}\left[\log \left(A(\omega)[|t|+2]^{d}\right)\right]^{2 d} \frac{d t}{\left(\frac{1}{2}+|t|\right)^{k+1}} \\
& \ll \int_{T}^{\infty}\left[A t^{d} Q^{d}\right]^{\frac{1}{2}}\left[\log \left(A t^{d} Q^{d}\right)\right]^{2 d} \frac{d t}{\left(\frac{1}{2}+|t|\right)^{k+1}} \\
& \ll\left(A Q^{d}\right)^{\frac{1}{2}} \log \left(A Q^{d}\right)^{2 d} d^{2 d} \int_{T}^{\infty} t^{\frac{d}{2}-k-1}(\log t)^{2 d} d t \\
& \ll\left(A Q^{d}\right)^{\frac{1}{2}} \log \left(A Q^{d}\right)^{2 d} d^{2 d} \frac{(\log T)^{2 d}}{\left(k-\frac{d}{2}\right) T^{k-\frac{d}{2}}}
\end{aligned}
$$

Therefore,

$$
\begin{aligned}
\sum_{q \leq Q} \sum_{\omega} * \int_{T}^{\infty} \frac{\left|L\left(\frac{1}{2}+i t, \omega\right)\right|^{2} d t}{\left(\frac{1}{2}+|t|\right)^{k+1}} & \ll \sum_{q \leq Q} \sum_{\omega} *\left(A Q^{d}\right)^{\frac{1}{2}} \log \left(A Q^{d}\right)^{2 d} d^{2 d} \frac{(\log T)^{2 d}}{\left(k-\frac{d}{2}\right) T^{k-\frac{d}{2}}} \\
& \ll A^{\frac{1}{2}} Q^{\frac{d}{2}+2} \log \left(A Q^{d}\right)^{2 d} d^{2 d} \frac{(\log T)^{2 d}}{\left(k-\frac{d}{2}\right) T^{k-\frac{d}{2}}} .
\end{aligned}
$$

The proof is similar for the integral in the range $(-\infty,-T]$.

For the estimate of

$$
\sum_{q \leq Q} \sum_{\omega}^{*} \int_{T}^{\infty} \frac{\left|L^{\prime}\left(\frac{1}{2}+i t, \omega\right)\right|^{2} d t}{(|t|+2)^{k+1}}
$$

Proposition 3.6 implies that

$$
\int_{T}^{\infty} \frac{\left|L\left(\frac{1}{2}+i t, \omega\right)\right|^{2} d t}{(|t|+2)^{k+1}} \ll \int_{T}^{\infty} 2^{d}\left[A(\omega)(|t|+2)^{d}\right]^{\frac{1}{2}}\left[\log \left(A(\omega)[|t|+2]^{d}\right)\right]^{2 d+2} \frac{d t}{\left(\frac{1}{2}+|t|\right)^{k+1}}
$$

which is two factors of $\log \left(A(\omega)[|t|+2]^{d}\right)$ more than in the estimate for $L$. As such,

$$
\sum_{q \leq Q} \sum_{\omega}^{*} \int_{T}^{\infty} \frac{\left|L^{\prime}\left(\frac{1}{2}+i t, \omega\right)\right|^{2} d t}{\left(\frac{1}{2}+|t|\right)^{k+1}} \ll A^{\frac{1}{2}} Q^{\frac{d}{2}+2} \log \left(A Q^{d}\right)^{2 d+2} d^{2 d+1} \frac{(\log T)^{2 d+2}}{\left(k-\frac{d}{2}\right) T^{k-\frac{d}{2}}} .
$$

The proof is similar for the integral in the range $(-\infty,-T]$.

\subsection{Mean Value Estimates}

We now estimate the remainder of the $S_{4}^{\prime}$ and $S_{4}^{\prime \prime}$ summations.

Lemma 3.9. With $U=\left(A Q^{d} T^{d}\right)^{\frac{1}{2}}$, when $T>1$,

1. $\sum_{q \leq Q} \sum_{\omega}^{*} \int_{-T}^{T} \frac{\left|L\left(\frac{1}{2}+i t, \omega\right)\right|^{2} d t}{\left(\frac{1}{2}+|t|\right)^{k+1}} \ll(d !)^{2} T(\log U)^{3 d-1}\left(U+Q^{2}\right)$.

2. $\sum_{q \leq Q} \sum_{\omega}^{*} \int_{-T}^{T} \frac{\left|L^{\prime}\left(\frac{1}{2}+i t, \omega\right)\right|^{2} d t}{\left(\frac{1}{2}+|t|\right)^{k+1}} \ll(d !)^{2} T(1+\log T)(\log U)^{3 d+1}\left(U+Q^{2}\right)$.

This combined with Lemma 3.8, upon taking $T=Q^{\frac{\epsilon}{d}}$ (which implies that $T>1$ as $Q>1$ ) results in the following estimate for $S_{4}$. 
Proposition 3.10. If $0<\epsilon<\frac{1}{4}, Q>1$, and $k>\frac{d}{2}\left(1+\frac{d}{\epsilon}\right)$, with

$$
F_{1}=d^{2 d} Q^{2}(\log Q)^{4 d}(\epsilon d \log A)^{2 d} A^{\frac{1}{2}}
$$

and

$$
F_{2}=(d !)^{2}\left(A^{\frac{1}{2}} Q^{\frac{d}{2}+\epsilon\left(\frac{1}{2}+\frac{1}{d}\right)}+Q^{2+\frac{\epsilon}{d}}\right)\left(\log A Q^{\epsilon+d}\right)^{3 d-1},
$$

1. $S_{4}^{\prime} \ll F_{1}+F_{2}$.

2. $S_{4}^{\prime \prime} \ll d(\log Q)^{2}(\epsilon d \log A)^{2} F_{1}+\left(1+\frac{\epsilon}{2} \log Q\right)\left(\log A Q^{\epsilon+d}\right)^{2} F_{2}$.

We begin with the estimate of $L(s, \omega)$, and then estimate $L^{\prime}(s, \omega)$ in a similar manner. We use the following lemma in both proofs.

Lemma 3.11. For any positive parameters $U$ and $V$, with $C_{1}=-\frac{1}{2}-\frac{1}{\log V}$,

$$
L(s, \omega)=\sum_{n=1}^{\infty} a_{n} \omega(n) n^{-s}=\sum_{n=1}^{\infty} a_{n} \omega(n) e^{-\frac{n}{U}} n^{-s}-\frac{1}{2 \pi i} \int_{\left(C_{1}\right)} L(s+w, \omega) U^{w} \Gamma(w) d w .
$$

We devote $\S 3.3 .1$ to the proof of Lemma 3.9 part 1, and $\S 3.3 .2$ to the proof of Lemma 3.9 part 2 .

\subsubsection{Mean Value Estimates for $L$}

In this section we prove Lemma 3.9 part 1. From Lemma 3.11 and the functional equation (0.4.3) for $L(s+w, \omega)$, with $C_{1}=-\frac{1}{2}-\frac{1}{\log V}$,

$$
\begin{aligned}
L(s, \omega)= & \sum_{n=1}^{\infty} a_{n} \omega(n) e^{-\frac{n}{U}} n^{-s}-\frac{1}{2 \pi i} \int_{\left(C_{1}\right)} \Theta(s+w, \omega) L(1-s-w, \bar{\omega}) U^{w} \Gamma(w) d w \\
= & \sum_{n=1}^{\infty} a_{n} \omega(n) e^{-\frac{n}{U}} n^{-s}-\frac{1}{2 \pi i} \int_{\left(C_{1}\right)} \Theta(s+w, \omega) \sum_{n>U} a_{n} \bar{\omega}(n) n^{-1+s+w} U^{w} \Gamma(w) d w \\
& \quad-\frac{1}{2 \pi i} \int_{\left(C_{2}\right)} \Theta(s+w, \omega) \sum_{n \leq U} a_{n} \bar{\omega}(n) n^{-1+s+w} U^{w} \Gamma(w) d w \\
= & A_{1}(s, \omega)+A_{2}(s, \omega)+A_{3}(s, \omega)
\end{aligned}
$$

where we have moved the final integral to the line $C_{2}=-(\log V)^{-1}$. By the Cauchy-Schwarz inequality,

$$
\sum_{q \leq Q} \sum_{\omega}^{*} \int_{-T}^{T} \frac{\left|L\left(\frac{1}{2}+i t\right)\right|^{2} d t}{\left(\frac{1}{2}+|t|\right)^{k+1}} \ll B_{1}+B_{2}+B_{3}
$$

where $B_{i}=\sum_{q \leq Q} \sum_{\omega}^{*} \int_{-T}^{T}\left|A_{i}\left(\frac{1}{2}+i t, \omega\right)\right|^{2} d t$.

We will estimate each of the three summands separately, achieving the following estimates with $U=V=$ $\left(A Q^{d} T^{d}\right)^{\frac{1}{2}}$.

Claim 3.12. $B_{1} \ll(d !)^{2}(\log U)^{2 d-1}\left(U+Q^{2}\right)$.

Claim 3.13. $B_{2} \ll(d !)^{2}(\log U)^{3 d-1}\left(U+Q^{2} T\right)$.

Claim 3.14. $B_{3} \ll(d !)^{2}(\log U)^{2 d-1}\left(1+Q^{2} U^{-1} T\right)$.

First we will prove Claim 3.13, then Claim 3.12, and finally Claim 3.14.

Proof of Claim 3.13. Let $I(U)=\sum_{n>U} a_{n} \bar{\omega}(n) n^{-\frac{1}{2}+C_{1}+i(t+y)}$. Break up the integral into two parts, $B_{2} \ll$ $B_{2}^{\prime}+B_{2}^{\prime \prime}$ where

$$
B_{2}^{\prime}=\sum_{q \leq Q} \sum_{\omega}^{*} \int_{-T}^{T}\left|\int_{|y| \leq \log U} \Theta\left(\frac{1}{2}+C_{1}+i(t+y), \omega\right) I(U) U^{C_{1}+i y} \Gamma\left(C_{1}+i y\right) d y\right|^{2} d t
$$


and $B_{2}^{\prime \prime}$ is identical, except that the inner integral is over $|y|>\log U$.

We first show that $B_{2}^{\prime} \ll(d !)^{2} U(\log U)^{3 d-1}\left(1+Q^{2} T U^{-1}\right)$. In the region $|y| \leq \log U, \Gamma\left(C_{1}+i y\right)$ has no poles, so is bounded by (0.4.4). By (0.4.5), $\Theta\left(-\frac{1}{\log U}+i(t+y), \omega\right) \ll U(\log U)^{\frac{d}{2}}$. By Theorem 3.2

$$
\begin{aligned}
B_{2}^{\prime} & \ll U(\log U)^{d} \int_{|y| \leq \log U} \sum_{q \leq Q} \sum_{\omega}^{*} \int_{-T}^{T}\left|\sum_{n>U} a_{n} \bar{\omega}(n) n^{-1-\frac{1}{\log U}+i(t+y)}\right|^{2} d t d y \\
& \ll U(\log U)^{d+1} \sum_{n>U}\left|a_{n}\right|^{2} n^{-2-\frac{2}{\log U}}\left(n+Q^{2} T\right) \\
& \ll(d !)^{2} U(\log U)^{d+1} \sum_{n>U}(\log n)^{2 d-2} n^{-2-\frac{2}{\log U}}\left(n+Q^{2} T\right) \\
& \ll(d !)^{2} U(\log U)^{3 d-1}\left(1+Q^{2} T U^{-1}\right) .
\end{aligned}
$$

We now show that $B_{2}^{\prime \prime} \ll(d !)^{2} T U^{\frac{3}{2}-\pi}(\log U)^{2 d-2}\left(1+Q^{2} U^{-1}\right)$, which is smaller than the desired bound as $U>1$. We concentrate on the integral over $|y|>\log U$, with $C_{1}=-\frac{1}{2}-(\log U)^{-1}$; here $\Gamma\left(C_{1}+i y\right) \ll$ $U^{-\frac{\pi}{2}}(\log U)^{-1}$ by $(0.4 .4)$. This integral is

$$
\begin{aligned}
& \int_{|y|>\log U} \Theta\left(-\frac{1}{\log U}+i(t+y), \omega\right) I(U) U^{-\frac{1}{2}-\frac{1}{\log U}+i y} \Gamma\left(-\frac{1}{2}-\frac{1}{\log U}+i y\right) d y \\
& \ll U^{-\frac{1}{2}-\frac{1}{\log U}} \int_{|y|>\log U} \frac{\left[A(\omega)(|t+y|+2)^{d}\right]^{1+\frac{1}{\log U}}}{e^{\frac{1}{2} \pi|y|}|y|^{1+\frac{1}{\log U}}}|I(U)| d y \\
& \ll U^{-\frac{1}{2}} \int_{|y|>\log U} \frac{\left(\left[A Q^{d} T^{d}\right]^{1+\frac{1}{\log U}}+y^{d\left(\frac{1}{2}+\frac{1}{\log U}\right)}\right)}{e^{\frac{1}{2} \pi|y|}|y|^{1+\frac{1}{\log U}}}|I(U)| d y \\
& \ll U^{-\frac{1}{2}} \int_{|y|>\log U} \frac{\left(U^{2}+y^{d\left(\frac{1}{2}+\frac{1}{\log U}\right)}\right)}{e^{\frac{1}{2} \pi|y|}|y|^{1+\frac{1}{\log U}}}|I(U)| d y
\end{aligned}
$$

where we have used Stirling's approximation of $\Theta$ as in (0.4.5). Letting

$$
\alpha_{1}=U^{\frac{3}{2}} \int_{|y|>\log U} e^{-\frac{1}{2} \pi|y|}|y|^{-1-\frac{1}{\log U}}|I(U)| d y \quad \text { and } \quad \alpha_{2}=U^{-\frac{1}{2}} \int_{|y|>\log U} \frac{y^{d\left(\frac{1}{2}+\frac{1}{\log U}\right)}}{e^{\frac{1}{2} \pi|y|}|y|^{1+\frac{1}{\log U}}}|I(U)| d y,
$$

$B_{2}^{\prime \prime} \ll \beta_{1}+\beta_{2}$ where $\beta_{i}=\sum_{q \leq Q} \sum_{\omega}^{*} \int_{-T}^{T} \alpha_{i} d t$.

By the Cauchy-Schwarz inequality,

$$
\beta_{1} \ll U^{\frac{3}{2}} \sum_{q \leq Q} \sum_{\omega}^{*} \int_{-T}^{T}\left(\int_{|y|>\log U} e^{-\pi|y|} d y\right)\left(\left.\left.\int_{|y|>\log U}|| y\right|^{-1-\frac{1}{\log U}} I(U)\right|^{2} d y\right) d t .
$$

The first integral with respect to $y$ is $O\left(U^{-\pi}\right)$. So,

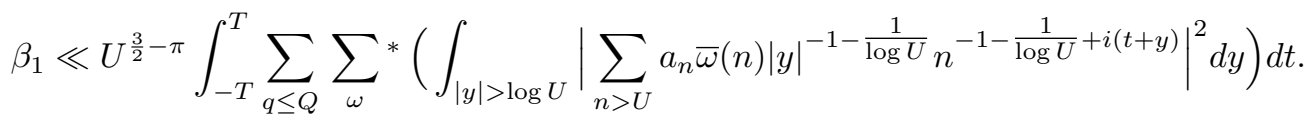


We now apply Theorem 3.2 to the integral with respect to $y$, mimicking the proof of Corollary 3.3 .

$$
\begin{aligned}
\beta_{1} & \ll U^{\frac{3}{2}-\pi} \int_{-T}^{T} \sum_{j>\log U} \sum_{q \leq Q} \sum_{\omega} *\left(\left.\left.\int_{-(j+1)}^{-j}\left|\sum_{n>U} a_{n} \bar{\omega}(n)\right| y\right|^{-1-\frac{1}{\log U}} n^{-1-\frac{1}{\log U}+i(t+y)}\right|^{2} d y\right. \\
& \left.+\left.\left.\int_{j}^{j+1}\left|\sum_{n>U} a_{n} \bar{\omega}(n)\right| y\right|^{-1-\frac{1}{\log U}} n^{-1-\frac{1}{\log U}+i(t+y)}\right|^{2} d y\right) d t \\
& \ll U^{\frac{3}{2}-\pi} \int_{-T}^{T} \sum_{j>\log U}^{\infty} \frac{1}{j^{2+\frac{2}{\log U}}} \sum_{n>U} \frac{\left|a_{n}\right|^{2}}{n^{2+\frac{2}{\log U}}}\left(n+Q^{2}(j+1)\right) d t \\
& \ll(d !)^{2} T U^{\frac{3}{2}-\pi} \sum_{n>U}(\log n)^{2 d-2}\left(n^{-1-\frac{2}{\log U}}+n^{-2-\frac{2}{\log U}} Q^{2}\right) \\
& \ll(d !)^{2} T U^{\frac{3}{2}-\pi}(\log U)^{2 d-2}\left(1+Q^{2} U^{-1}\right) .
\end{aligned}
$$

The estimate of $\beta_{2}$ is similar. By the Cauchy-Schwarz inequality,

$$
\beta_{2} \ll U^{-\frac{1}{2}} \sum_{q \leq Q} \sum_{\omega}^{*} \int_{-T}^{T}\left(\int_{|y|>\log U} \frac{y^{d\left(\frac{1}{2}+\frac{1}{\log U}\right)}}{e^{\pi|y|}} d y\right)\left(\left.\left.\int_{|y|>\log U}|| y\right|^{-1-\frac{1}{\log U}} I(U)\right|^{2} d y\right) d t .
$$

The first integral with respect to $y$ is $O\left((\log U)^{\frac{d}{2}} U^{-\pi}\right)$. The remainder of the calculation is exactly as above. We get

$$
\begin{aligned}
\beta_{2} & \ll(\log U)^{\frac{d}{2}} U^{-\pi-\frac{1}{2}} \sum_{q \leq Q} \sum_{\omega}^{*} \int_{-T}^{T}\left(\left.\left.\int_{|y|>\log U}|| y\right|^{-1-\frac{1}{\log U}} I(U)\right|^{2} d y\right) d t \\
& \ll(d !)^{2} T U^{-\pi-\frac{1}{2}}(\log U)^{\frac{3}{2} d-2}\left(1+Q^{2} U^{-1}\right) .
\end{aligned}
$$

Proof of Claim 3.12. By definition,

$$
B_{1}=\sum_{q \leq Q} \sum_{\omega}^{*} \int_{-T}^{T}\left|\sum_{n=1}^{\infty} a_{n} \omega(n) e^{-\frac{n}{U}} n^{-\frac{1}{2}-i t}\right|^{2} d t
$$

and by Corollary 3.3

$$
B_{1} \ll \sum_{n=1}^{\infty}\left|a_{n}\right|^{2} e^{-\frac{2 n}{U}} n^{-1}\left(n+Q^{2}\right) \ll(d !)^{2} \sum_{n=1}^{\infty}(\log n)^{2 d-2} e^{-\frac{2 n}{U}} n^{-1}\left(n+Q^{2}\right) .
$$

We break this sum up into the sum over $n \leq U$ and then the sum $n>U$. In the first range the summation is of the order

$$
\begin{aligned}
(d !)^{2}(\log U)^{2 d-2} \sum_{n \leq U} e^{-\frac{2 n}{U}}\left(1+\frac{Q^{2}}{n}\right) & \ll(d !)^{2}(\log U)^{2 d-2}\left(U+Q^{2} \sum_{n \leq U} \frac{1}{n}\right) \\
& \ll(d !)^{2}(\log U)^{2 d-1}\left(U+Q^{2}\right) .
\end{aligned}
$$

In the range $n>U, e^{-\frac{2 n}{U}} \ll n^{-1-\frac{2}{\log U}}$, so the bound $(d !)^{2}(\log U)^{2 d-2}\left(U+Q^{2}\right) U^{-1}$ for the summations in $B_{2}$ suffices. Since $U>1$,

$$
B_{1} \ll(d !)^{2}(\log U)^{2 d-1}\left(U+Q^{2}\right)\left(1+(\log U)^{-1} U^{-1}\right) \ll(d !)^{2}(\log U)^{2 d-1}\left(U+Q^{2}\right) .
$$


Proof of Claim 3.14. Let $I(U)=\sum_{n \leq U} a_{n} \bar{\omega}(n) n^{-\frac{1}{2}+C_{2}+i(t+y)}$. We have $B_{3} \ll B_{3}^{\prime}+B_{3}^{\prime \prime}$ where

$$
B_{3}^{\prime}=\sum_{q \leq Q} \sum_{\omega}^{*} \int_{-T}^{T}\left|\int_{|y|<\log U} \Theta\left(\frac{1}{2}+C_{2}+i(t+y), \omega\right) I(U) U^{C_{2}+i y} \Gamma\left(C_{2}+i y\right) d y\right|^{2} d t
$$

where $B_{3}^{\prime \prime}$ is the sum with the $y$ integral restricted to the range $|y|>\log U$. With $C_{2}=-(\log U)^{-1}$ using (0.4.5), $\Theta\left(\frac{1}{2}-\frac{1}{\log U}+i(t+y), \omega\right) \ll\left[A(w)(|t+y|+2)^{d}\right]^{\frac{1}{\log U}}$ is bounded. Also, (0.4.4) implies that $\Gamma\left(-\frac{1}{\log U}+i y\right) \ll U^{-\frac{\pi}{2}}(\log U)^{-\frac{1}{2}}$ when $|y|>\log U$. Therefore, using the estimate derived from Stirling's formula for $\Gamma\left(C_{2}+i y\right)$, we get

$$
B_{3}^{\prime \prime} \ll \sum_{q \leq Q} \sum_{\omega}^{*} \int_{-T}^{T} \int_{|y|>\log U} e^{-\pi|y|}|y|^{-1-\frac{2}{\log U}}|I(U)|^{2} d y d t .
$$

After switching the order of integration and using Theorem 3.2 to estimate the sums and the inner integral with respect to $t$, as the remaining integral with respect to $y$ is $O\left(U^{-\pi} \log U\right)$,

$$
B_{3}^{\prime \prime} \ll U^{-\pi} \sum_{n \leq U}\left|a_{n}\right|^{2} n^{-1-\frac{2}{\log U}}\left(n+Q^{2} T\right) \ll(d !)^{2} U^{-\pi}(\log U)^{2 d-2}\left(1+Q^{2} U^{-1} T\right) .
$$

In the region $|y| \leq \log U, \Theta\left(\frac{1}{2}-\frac{1}{\log U}+i(t+y), \omega\right)$ is bounded, and by (0.4.4), $\Gamma\left(-\frac{1}{\log U}+i y\right)$ is bounded as well. Therefore

$$
B_{3}^{\prime} \ll \int_{|y| \leq \log U} \sum_{q \leq Q} \sum_{\omega}^{*} \int_{-T}^{T}|I(U)|^{2} d t d y .
$$

Using Theorem 3.2

$$
B_{3}^{\prime} \ll(\log U) \sum_{n \leq U}\left|a_{n}\right|^{2} n^{-1-\frac{2}{\log U}}\left(n+Q^{2} T\right) \ll(d !)^{2}(\log U)^{2 d-1}\left(1+Q^{2} U^{-1} T\right) .
$$

\subsubsection{Mean Value Estimates for $L^{\prime}$}

In this section we prove Lemma 3.9 part 2. We begin with Lemma 3.11

$$
L(s, \omega)=\sum_{n=1}^{\infty} a_{n} \omega(n) e^{-\frac{n}{U}} n^{-s}-\frac{1}{2 \pi i} \int_{\left(C_{1}\right)} L(s+w, \omega) U^{w} \Gamma(w) d w
$$

and differentiate with respect to $s$ to get

$$
L^{\prime}(s, \omega)=-\sum_{n=1}^{\infty} a_{n}(\log n) \omega(n) e^{-\frac{n}{U}} n^{-s}-\frac{1}{2 \pi i} \int_{\left(C_{1}\right)} L^{\prime}(s+w, \omega) U^{w} \Gamma(w) d w .
$$

Differentiating the functional equation (0.4.3) for $L, L^{\prime}(s, \omega)=-\Theta(s, \omega) L^{\prime}(1-s, \omega)+\Theta^{\prime}(s, \omega) L(1-s, \omega)$. We have $L^{\prime}(s, \omega)=\sum_{i=1}^{5} D_{i}(s, \omega)$ where 


$$
\begin{aligned}
& D_{1}=\sum_{n=1}^{\infty} a_{n}(\log n) \omega(n) e^{-\frac{n}{U}} n^{-s} \\
& D_{2}=-\frac{1}{2 \pi i} \int_{\left(C_{1}\right)} \Theta(s, \omega) \sum_{n>U} a_{n}(\log n) \bar{\omega}(n) n^{-1+s+w} U^{w} \Gamma(w) d w \\
& D_{3}=-\frac{1}{2 \pi i} \int_{\left(C_{1}\right)} \Theta^{\prime}(s, \omega) \sum_{n>U} a_{n} \bar{\omega}(n) n^{-1+s+w} U^{w} \Gamma(w) d w \\
& D_{4}=-\frac{1}{2 \pi i} \int_{\left(C_{2}\right)} \Theta(s, \omega) \sum_{n \leq U} a_{n}(\log n) \bar{\omega}(n) n^{-1+s+w} U^{w} \Gamma(w) d w \\
& D_{5}=-\frac{1}{2 \pi i} \int_{\left(C_{2}\right)} \Theta^{\prime}(s, \omega) \sum_{n \leq U} a_{n} \bar{\omega}(n) n^{-1+s+w} U^{w} \Gamma(w) d w
\end{aligned}
$$

with $C_{1}=-\frac{1}{2}-\frac{1}{\log V}$ and $C_{2}=-\frac{1}{\log V}$ are as in the $L$ estimates. By the Cauchy-Schwarz inequality,

$$
\sum_{q \leq Q} \sum_{\omega}^{*} \int_{-T}^{T} \frac{\left|L^{\prime}\left(\frac{1}{2}+i t, \omega\right)\right|^{2} d t}{(|t|+2)^{k+1}} \ll E_{1}+E_{2}+E_{3}+E_{4}+E_{5}
$$

where $E_{i}=\sum_{q \leq Q} \sum_{\omega}^{*} \int_{-T}^{T}\left|D_{i}\left(\frac{1}{2}+i t, \omega\right)^{2}\right|(|t|+2)^{-k-1} d t$ for $1 \leq i \leq 5$. After using the large sieve, the sums $E_{1}$ and $E_{2}$ are a magnification of $B_{1}$ and $B_{2}$, each by a factor of $(\log U)^{2}$. For $E_{4}$, as $n \leq U$ there is an additional factor of $(\log U)$ multiplied by the estimate of $B_{3}$. It remains to consider $E_{3}$ and $E_{5}$. By (0.4.6) $\frac{\Theta}{\Theta}^{\prime}(s+w, \omega) \ll d\left[\log (|y+t|+2)+\frac{1}{|s+w|}\right]$. Since $\Theta^{\prime}(s+w, \omega)=\Theta(s+w, \omega) \frac{\Theta^{\prime}}{\Theta}(s+w, \omega)$, this results in an additional factor of $d\left[\log (|y+t|+2)+\frac{1}{|s+w|}\right]$ in the estimates of $B_{2}$ and $B_{3}$. In $E_{3}, s+w=-\frac{1}{\log U}+i(t+y)$. For the portion of the integral where $|y|>\log U$, (corresponding to the $B_{2}^{\prime \prime}$ estimate) the second summand is bounded by $d(\log U)$ and the first logarithm term is $O(d \log T+d \log y)=O(d(\log T y))$ which results in a magnification of the integral $B_{2}^{\prime \prime}$ by a factor of $O(d \log U)$, since $T \leq U$. For the portion of the integral with $|y| \leq \log U$ (corresponding to the $B_{2}^{\prime}$ estimate) the additional term is easily seen to be $O(d \log U)$. Therefore, $E_{3}$ is a magnification of the estimate for $B_{2}$ by a factor of $O(d \log U)$.

For $E_{5}, s+w=\frac{1}{2}-\frac{1}{\log U}+i(t+y)$ and the estimates have an additional factor of $d[\log (|y+t|+2)+$ $\left.\left(\left|\frac{1}{2}-\frac{1}{\log U}+i(t+y)\right|\right)^{-1}\right]$. Since $U>1$ this second term is bounded. For the portion of the integral where $|y| \leq \log U$, (corresponding to the $B_{3}^{\prime}$ estimate) the first summand is bounded by $d(\log T+\log \log U)$. For the portion of the integral where $|y|>\log U$, (corresponding to the $B_{3}^{\prime \prime}$ estimate) there is an additional factor of $d \log (|t+y|+2)$ in the summation. Upon integrating, since $|t| \leq T \leq U$, this results in a magnification of $O(d \log U)$ over the $B_{3}$ estimate for the $E_{5}$ estimate.

\subsection{Combining the estimates}

We now prove Proposition 3.1. From Propositions 3.5 and 3.10 and equation (3.0.2) for $0<\epsilon<\frac{1}{4}$

$$
\begin{aligned}
\sum_{q \leq Q} \sum_{\omega}{ }^{*} \max _{y \leq x}\left|\tilde{\psi}_{k}(y, \omega)\right| & \ll x\left(S_{1}+S_{2}\right)+x^{\frac{1}{2}}\left(S_{3}+S_{4}\right) \\
\ll & \ll(\log x)^{(d+4)^{2}}\left(1+Q^{2} z^{-1}\right)+x^{\frac{1}{2}}(\log z)^{(d+4)^{2}}\left(z^{2}+Q^{2}\right) \\
& \quad+x^{\frac{1}{2}}\left(Q^{2}(\log Q)^{4 d+2}+Q^{\frac{d}{2}+\epsilon\left(\frac{1}{2}+\frac{1}{d}\right)}(\log Q)^{3 d+2}+Q^{2+\frac{\epsilon}{d}}(\log Q)^{3 d+2}\right) \\
& \ll(\log x)^{(d+4)^{2}}\left(x\left(1+Q^{2} z^{-1}\right)+x^{\frac{1}{2}}\left(z^{2}+Q^{2}\right)+x^{\frac{1}{2}}\left(Q^{2+\epsilon^{\prime}}+Q^{\frac{d}{2}+\epsilon^{\prime}}\right)\right)
\end{aligned}
$$

with $\epsilon^{\prime}=\left(\frac{1}{2}+\frac{1}{d}\right) \epsilon$, since $z \leq x$ and $1<Q \leq x$.

Choosing $z=Q(\log x)^{\gamma}$ this is of the order

$$
(\log x)^{(d+4)^{2}}\left(x+x Q(\log x)^{-\gamma}+x^{\frac{1}{2}} Q^{2}(\log x)^{2 \gamma}+x^{\frac{1}{2}} Q^{2+\epsilon^{\prime}}+x^{\frac{1}{2}} Q^{\frac{d}{2}+\epsilon^{\prime}}\right) .
$$


Therefore, if $\gamma>(d+4)^{2}+D$, then for all $Q$ in the range

$$
(\log x)^{\gamma} \leq Q \leq \min \left\{x^{\frac{1}{2}}(\log x)^{-\left(2 \gamma+\left((d+4)^{2}\right)+D\right)}, x^{\frac{1}{2}-\epsilon^{\prime}}, x^{\frac{1}{d-2}-\epsilon^{\prime}}\right\}
$$

it follows that

$$
\frac{1}{Q} \sum_{q \leq Q} \sum_{\omega}^{*} \max _{y \leq x}\left|\tilde{\psi}_{k}(y, \omega)\right| \ll \frac{x}{(\log x)^{D}} .
$$

This proves Proposition 3.1.

\section{Conclusion}

Propositions 2.1 and 3.1 imply the following.

Theorem 4.1. Let $K / M$ be a Galois extension of number fields, with group $G$ and let $C$ be a conjugacy class in $G$. Let $H$ be the largest abelian subgroup of $G$ such that $H \cap C \neq \emptyset$ and let $E$ be the fixed field of $H$. Let $\eta=\max \{[E: \mathbb{Q}]-2,2\}$ and for any $\epsilon>0$ let $Q=x^{\frac{1}{\eta}-\epsilon}$. Then for any $A>0$,

$$
\sum_{q \leq Q}^{\prime} \max _{(a, q)=1} \max _{y \leq x}\left|\psi(y, C, q, a)-\frac{|C|}{|G|} \frac{y}{\phi(q)}\right| \ll \frac{x}{(\log x)^{A}} .
$$

This is equivalent to Theorem 0.2. An immediate corollary is

Corollary 4.2. Let $K$ be a number field and let $\eta=\max \{[K: \mathbb{Q}]-2,2\}$ and for any $\epsilon>0$ let $Q=x^{\frac{1}{\eta}-\epsilon}$. Then for any $A>0$,

$$
\sum_{q \leq Q}^{\prime} \max _{(a, q)=1} \max _{y \leq x}\left|\psi(y, q, a)-\frac{y}{\phi(q)}\right| \ll \frac{x}{(\log x)^{A}} .
$$

\subsection{Refinements of the main theorem}

Writing $\eta=\max \{d-2,2\}$ with $d=n_{E}$, it is possible to replace $\eta$ by a smaller value by replacing $d$ by $d^{*}$ where

$$
d^{*}=\min _{H} \max _{\varpi}[G: H] \varpi(1)
$$

where the maximum is over irreducible characters $\varpi$ of $H$ and the minimum is over all subgroups $H$ of $G$ such that

1. $H \cap C \neq \emptyset$.

2. For every irreducible character $\varpi$ of $H$ and any non-trivial Dirichlet character $\chi$ the Artin $L$-function $L(s, \varpi \otimes \chi)$ is entire. (Here, if $q$ is the modulus of $\chi$ we consider $\varpi \otimes \chi$ as an element of $G \times \mathbb{Z} / q \mathbb{Z}$ as before.)

If the Artin Conjecture that the $L$-functions associated to all abelian twists of the non-trivial irreducible characters of $G=\operatorname{Gal}(K / M)$ are entire, then we can set $\eta=\max \{\delta, 2\}$ where $\delta=\max |\chi(1)-2|$ where the maximum is over $\chi \neq \chi_{0}$. A value of $\delta \leq 2$ results in the optimal bound of $Q^{\frac{1}{2}-\epsilon}$. The groups $G$ which satisfy $\delta \leq 2$ can be classified (cf. [9] Theorem 24.6).

Acknowledgement. We thank the referee for the helpful and detailed remarks on an earlier version of this paper. 


\title{
References
}

[1] Hideo Aramata. Über die Teilbarkeit der Dedekindschen Zetafunktionen. Proc. Imp. Acad., 9(2):31-34, 1933.

[2] Paul T. Bateman and Harold G. Diamond. Analytic number theory. World Scientific Publishing Co. Pte. Ltd., Hackensack, NJ, 2004. An introductory course.

[3] E. Bombieri. On the large sieve. Mathematika, 12:201-225, 1965.

[4] Enrico Bombieri. Le grand crible dans la théorie analytique des nombres. Astérisque, (18):103, 1987.

[5] Richard Brauer. On the zeta-functions of algebraic number fields. Amer. J. Math., 69:243-250, 1947.

[6] J.W. Cogdell. On Artin L-functions. www.math.ohio-state.edu/ cogdell.

[7] Harold Davenport. Multiplicative number theory, volume 74 of Graduate Texts in Mathematics. Springer-Verlag, New York, second edition, 1980. Revised by Hugh L. Montgomery.

[8] Max Deuring. Über den Tschebotareffschen Dichtigkeitssatz. Math. Ann., 110(1):414-415, 1935.

[9] Walter Feit. Characters of finite groups. W. A. Benjamin, Inc., New York-Amsterdam, 1967.

[10] P. X. Gallagher. Bombieri's mean value theorem. Mathematika, 15:1-6, 1968.

[11] G. H. Hardy and E. M. Wright. An introduction to the theory of numbers. Oxford, at the Clarendon Press, 1954. 3rd ed.

[12] Malcolm Harper and M. Ram Murty. Euclidean rings of algebraic integers. Canad. J. Math., 56(1):71-76, 2004.

[13] J. C. Lagarias and A. M. Odlyzko. Effective versions of the Chebotarev density theorem. In Algebraic number fields: L-functions and Galois properties (Proc. Sympos., Univ. Durham, Durham, 1975), pages 409-464. Academic Press, London, 1977.

[14] C. R. MacCluer. A reduction of the Čebotarev density theorem to the cyclic case. Acta Arith., 15:45-47, 1968.

[15] J. Martinet. Character theory and Artin L-functions. In Algebraic number fields: L-functions and Galois properties (Proc. Sympos., Univ. Durham, Durham, 1975), pages 1-87. Academic Press, London, 1977.

[16] M. Ram Murty. Problems in analytic number theory, volume 206 of Graduate Texts in Mathematics. Springer, New York, second edition, 2008. Readings in Mathematics.

[17] M. Ram Murty and V. Kumar Murty. A variant of the Bombieri-Vinogradov theorem. In Number theory (Montreal, Que., 1985), volume 7 of CMS Conf. Proc., pages 243-272. Amer. Math. Soc., Providence, RI, 1987.

[18] M. Ram Murty and Kathleen L. Petersen. The generalized Artin conjecture and arithmetic orbifolds. In Groups and symmetries, volume 47 of CRM Proc. Lecture Notes, pages 259-265. Amer. Math. Soc., Providence, RI, 2009.

[19] Władysław Narkiewicz. Elementary and analytic theory of algebraic numbers. PWN-Polish Scientific Publishers, Warsaw, 1974. Monografie Matematyczne, Tom 57.

[20] Hans Rademacher. On the Phragmén-Lindelöf theorem and some applications. Math. Z, 72:192-204, 1959/1960.

[21] K. Ramachandra. A simple proof of the mean fourth power estimate for $\zeta(1 / 2+i t)$ and $L(1 / 2+i t, X)$. Ann. Scuola Norm. Sup. Pisa Cl. Sci. (4), 1:81-97 (1975), 1974.

[22] H. M. Stark. Some effective cases of the Brauer-Siegel theorem. Invent. Math., 23:135-152, 1974.

[23] N. Tschebotareff. Die Bestimmung der Dichtigkeit einer Menge von Primzahlen, welche zu einer gegebenen Substitutionsklasse gehören. Math. Ann., 95(1):191-228, 1926.

[24] A. I. Vinogradov. The density hypothesis for Dirichlet L-series. Izv. Akad. Nauk SSSR Ser. Mat., 29:903-934, 1965.

\author{
M. RAm MurTy \\ Department of Mathematics and Statistics \\ Queen's University \\ Kingston, Ontario, K7L 3N6, Canada \\ email: murty@mast.queensu.ca \\ Kathleen L. Petersen \\ Department of Mathematics \\ Florida State University \\ Tallahassee, Florida, 32306, U.S.A. \\ email: petersen@math.fsu.edu
}

